\title{
PHYTOPLANKTON DYNAMICS IN A HIGHLY EUTROPHIC ESTUARY IN TROPICAL BRAZIL
}

\author{
Marilene Felipe Santiago ${ }^{1 *}$, Maria da Glória Gonçalves da Silva-Cunha ${ }^{I}$, Sigrid Neumann-Leitão ${ }^{I}$, \\ Kátia Muniz Pereira da Costa ${ }^{l}$, Gislayne Cristina Borges Palmeiral, \\ Fernando de Figueiredo Porto Neto ${ }^{2}$ and Fabiana Santana Nunes ${ }^{1}$
}

${ }^{1}$ Universidade Federal de Pernambuco - Departamento de Oceanografia (Av. Arquitetura s/n, Cidade Universitária, 50 601-910 Recife, PE, Brasil)

${ }^{2}$ Universidade Federal Rural de Pernambuco - Departamento de Zootecnia (Rua Dom Manoel de Medeiros, s/n, Dois Irmãos, 52171-900 Recife, PE, Brasil)

*Corresponding author: marilenesantiago@yahoo.com.br

\section{A B S T R A C T}

\begin{abstract}
The port of Recife in northeastern Brazil is an important ecological and social area but little is known about its environmental quality. Observations, sampling and measurements of phytoplankton were performed during spring and neap tides in the dry (January-February, 2005) and rainy (June, 2005) seasons to assess the environmental quality of the port of Recife. The area had chlorophyll- $a$ concentrations ranging from 3.30 to $54.40 \mathrm{mg} \mathrm{m}^{-3}$, the highest values occurring at low tide during the dry season. A total of 129 species were identified: 53 of them in the dry season and 97 in the rainy season. Diatoms were the most diverse group and comprised $75.47 \%$ of the phytoplankton collected in the dry season and $60.82 \%$ of those collected in the rainy season. The dry season was characterized by Coscinodiscus sp. and Helicotheca tamesis; the rainy season by Oscillatoria sp. and Coscinodiscus centralis. Species diversity indices varied from 1.06 to 3.74 bits cel $^{-1}$. Low indices were related to the dominance of Helicotheca tamesis, Coscinodiscus centralis, Coscinodiscus sp. and Aulacoseira granulata. Seasonal forcing, rather than the estuarine flux, determined the phytoplankton community structure. The area is exposed to seasonally varying negative impacts due to metropolitan degradation and the high level of eutrophication.
\end{abstract}

\section{RESUMO}

Para avaliar a qualidade ambiental Porto do Recife (Nordeste do Brasil), uma área ecológica e socialmente importante mas pouco investigada, observações, medições e amostragem do fitoplâncton foram realizadas durante as marés de sizígia e quadratura no período de estiagem (janeiro-fevereiro / 2005) e chuvoso (junho/2005). A área apresentou concentração de clorofila $a$ variando de 3,30 a $54,40 \mathrm{mg} \mathrm{m}^{-3}$, com maiores valores registrados durante a maré baixa no período de estiagem. Um total de 129 espécies foi identificada com 53 espécies durante o período de estiagem e 97 espécies no chuvoso. As diatomáceas formaram o grupo mais diverso, com 75,47\% no período de estiagem e $60,82 \%$ no chuvoso. Coscinodiscus sp. e Helicotheca tamesis caracterizaram o período de estiagem e Oscillatoria sp. e Coscinodiscus centralis o período chuvoso. A diversidade de espécie variou de

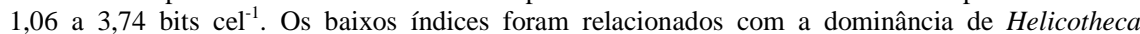
tamesis, Coscinodiscus centralis, Coscinodiscus sp. e Aulacoseira granulata. A sazonalidade determinou a estrutura da comunidade fitoplanctônica, em vez do fluxo estuarino. A área é exposta sazonalmente a vários impactos negativos, devido à degradação metropolitana e ao elevado nível de eutrofização.

Descriptors: Phytoplankton, Port, estuary, Diurnal variation, Tropical. Descritores: Fitoplâncton, Porto, Estuário, Variação diurnal, Tropical.

\section{INTRODUCTION}

The Pina Basin Estuaray is located in the metropolitan area of Recife in Pernambuco State, northeastern Brazil. As with many other tropical coastal areas, it is threatened by the diverse human uses of this body of water and the land areas that drain into it. Consequently, the port basin presents a multitude of environmental problems. The most common problems are degraded natural habitats, declining plant and animal populations, diminishing fish and shellfish harvests and impaired water quality.

This coastal area is of great ecological, economic and social interest. It is also a highly variable system, where changes in the water 
circulation patterns and fluctuations of input influences (e.g., Capibaribe River, Beberibe River, Tejipió River, sewage flow) induce high temporal variability on scales ranging from hours to seasons. This variability may be reflected in population dynamics, especially those of phytoplankton populations thriving in coastal systems.

Phytoplankton species can be used as a diagnostic tool in determining ecosystem quality (MAGUIRE, 1973; RESH, UNZICKER, 1975; DAY JR. et al., 1989). Previous research has identified biotic and abiotic factors that regulate primary productivity and has developed models that describe phytoplankton growth dynamics under specific environmental conditions (BAIRD et al., 2001).

Phytoplankton communities are highly complex multispecies communities in terms of their diversity and dynamics. Succession shifts in phytoplankton community structure are primarily due to changes in environmental variables (e.g., degree or type of nutrient limitation) and/or shifts in higher trophic levels (e.g., microzooplankton to mesozooplankton) (MILLER et al., 1995; GILBERT, 1998; LEWITUS et al., 1998; RIEGMAN; NOORDELOOS, 1998; RABOUILLE et al., 2001; FERNANDES; BRANDINI, 2004).

The influence of environmental variables and preferential grazing by herbivores on phytoplankton community composition is not well understood, particularly in harbor areas under extremely impacted influx. Identifying the ecological variables that regulate phytoplankton community structure is essential for facilitating the elaboration of broad hypotheses of pervasive environmental issues, such as eutrophication and harmful algal blooms (SMAYDA, 1997; RIEGMAN; FLAMELING; NOORDELOOS 1998; MAFRA JUNIOR et al., 2006). Since one of the most important factors affecting variability in the port of Recife and its basin is the tide, accurate estimates of the plankton requires sampling over a $24 \mathrm{~h}$ period (McLUSKY; ELLIOTT, 2004).

One question addressed in this study is whether the abiotic phenomena of the port basin of Recife (e.g., circulation patterns, tides) and water condition are related to identifiable phytoplankton assemblages or abundance patterns.

Despite the ecological and social importance of the port of Recife, few investigations of phytoplankton composition have been conducted in the harbor or in nearby areas (FEITOSA; PASSAVANTE, 1990; 1991/1993; FEITOSA et al., 1999; NASCIMENTO et al., 2003).

Many studies have been dedicated to the temporal variability of phytoplankton densities in marine systems (SOROKIN, 1995), but few data are available on the dynamics of tropical harbor phytoplankton communities.

The main objective of the present study was to study temporal changes of phytoplankton and hydrographic parameters in the highly polluted port basin of Recife in northeastern Brazil.

\section{Study Area}

The Port Basin Estuary is located in Recife, the capital of Pernambuco State, Brazil. Recife is the main urban center of northeastern Brazil and lies partly on the mainland and partly on the island. Dissected by waterways, it is often called the "Brazilian Venice" (Fig. 1).

This area has a hot and humid tropical climate and is categorized as Group A on the Köppen Scale. Annual average air and water temperatures are around $25^{\circ} \mathrm{C}$, the annual minimum being $24^{\circ} \mathrm{C}$ and the maximum $31^{\circ} \mathrm{C}$. Relative humidity varies from $80 \%$ to $90 \%$ and annual rainfall ranges from $1760 \mathrm{~mm}$ to 2270 mm, with $80 \%$ of it occurring between April and July (the rainy season). The dominant wind is from the southeast (ARAGÃO, 2004).

The maximum tidal height (during extreme spring tides) at the port is around $3 \mathrm{~m}$, with an annual high tide average of $2.6 \mathrm{~m}$ during spring tides and 1.6 $\mathrm{m}$ during neap tides. There are two natural access channels to the port. The main access channel, South Channel, is approximately $260 \mathrm{~m}$ wide and $3.4 \mathrm{~km}$ long with a depth of $10.5 \mathrm{~m}$. The North Channel, only used by smaller vessels, is narrower, being approximately $1.0 \mathrm{~km}$ long and $6.5 \mathrm{~m}$ deep. The basin constitutes a shallow, restricted environment that receives large quantities of untreated sewage and is, therefore, polluted, eutrophic and hypoxic.

\section{Material and Methods}

Sampling was carried out at one fixed station located at the confluence of the port of Recife, the Capibaribe River and the Pina Basin (08 $04^{\prime} 01^{\prime \prime} S$ and $34^{\circ} 52^{\prime} 06^{\prime W}$ ), called the Port basin (Fig. 1). Sampling was conducted on two consecutive days during the dry season (January 25-26 and February 02-03, 2005) and the rainy season (June 07-08 and 14-15, 2005) at spring and neap tides during the diurnal high, low, flood and ebb tides.

The following hydrological data were collected concurrently with a Nansen bottle: $\mathrm{pH}$ (Hanna 8417 pHmeter); dissolved oxygen (Winkler method; GRASSHOFF et al., 1983); dissolved oxygen saturation (UNESCO, 1973); nutrients, including nitrites, nitrates, phosphates and silicate (STRICKLAND; PARSONS, 1972; GRASSHOFF et 
al., 1983) and suspended particulate material (BAUMGARTEN et al., 1996). The following parameters were measured in situ : water temperature (Hanna digital thermometer), salinity (refractometer) and water transparency (Secchi disc). Chlorophyll- $a$ was collected with a 1 liter Van Dorn bottle at the surface and measured using a Micronal B280 spectrophotometer (PARSONS; STRICKLAND, 1963; WETZEL; LINKENS, 1991).

Phytoplankton sampling was conducted with a plankton net (mesh size $64 \mu \mathrm{m})$ fitted with a flowmeter. Three-minute horizontal surface hauls were carried out. After collection, samples were preserved in a $4 \%$ buffered formaldehyde/seawater solution (NEWELL; NEWELL, 1963).

In the laboratory, $300 \mathrm{~mL}$ samples were homogenized and a $1 \mathrm{~mL}$ subsample was analyzed under a compound microscope. Each cell, colony or filamentous alga was counted as an individual and expressed as relative abundance (\%). Identification was based on the phytoplankton literature (PÉRAGALLO; PÉRAGALLO, 1897-1908;
HUSTEDT, 1930-1966; CUPP, 1943; DESIKACHARY, 1959; SOURNIA, 1967; 1986; PRESCOTT, 1975; PARRA et al., 1982; TOMAS, 1996; KOMÁREK; ANAGNOSTIDIS, 2000).

The Shannon index was used to estimate the community diversity (SHANNON, 1948).

Cluster analysis of the sample-species data matrix was also performed, using the Bray \& Curtis method. The Weighted Pair Group Method, using arithmetic averages, was the link method used for the dendrograms (WPGMA) (LEGENDRE; LEGENDRE, 1998). A cophenetic value matrix was applied to test the adequacy of fit of the cluster analysis (ROHLF; FISHER, 1968). A Principal Component Analysis was computed based on a matrix formed by richness, diversity, chlorophyll- $a$ and hydrological data. Data analyses were carried out using the Numerical Taxonomy and Multivariate Analyses System (NTSYS ver. 1.30, Metagraphics Software Corporation, California, USA, 1987).
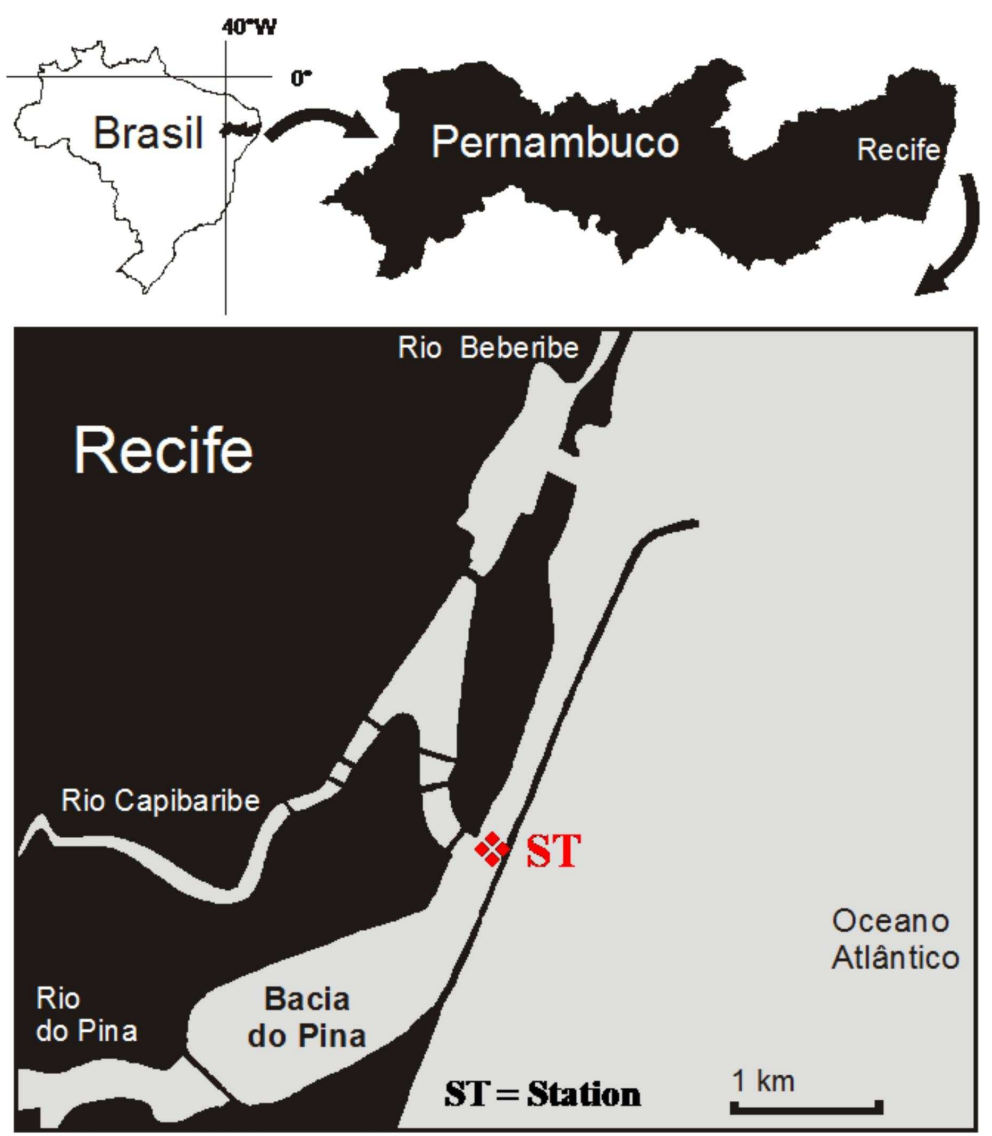

Fig. 1. Study area and sampling station (ST) in the Port Basin of Recife (Pernambuco, Brazil). 
A non-parametric Kruskal-Wallis test was applied (BioEstat 3.0) to test significant differences $(\mathrm{p}<0.05)$ between dry and rainy seasons and between tides, based on the physicochemical and biological parameters.

\section{Results}

\section{Physical Environment}

No significant difference $(p>0.5)$ in the abiotic parameters (water temperature, salinity, transparence, particulate organic material, $\mathrm{pH}$, dissolved oxygen, dissolved oxygen saturation tax, nitrite+nitrate, phosphate and silicate) was found between spring and neap tides. Water temperature varied between $27^{\circ} \mathrm{C}$ and $32.6^{\circ} \mathrm{C}$, the higher values being recorded in the late morning and early afternoon when the sunlight was more intense. Salinity varied between 21 and 36 during the dry season and ranged from 1 to 8 during the rainy season (Figs 2A, B; Figs $3 \mathrm{~A}, \mathrm{~B})$. Water transparency varied from $0.1 \mathrm{~m}$ to $1.0 \mathrm{~m}$ and higher values were recorded during the flood tide of the spring and neap tides in both seasons (Figs 2C, D; Figs 3C, D). The $\mathrm{pH}$ was $>7.0$ the larger part of the time. In general, temperature, salinity, transparency and $\mathrm{pH}$ were significantly higher during the dry season $(\mathrm{p}<0.05)$
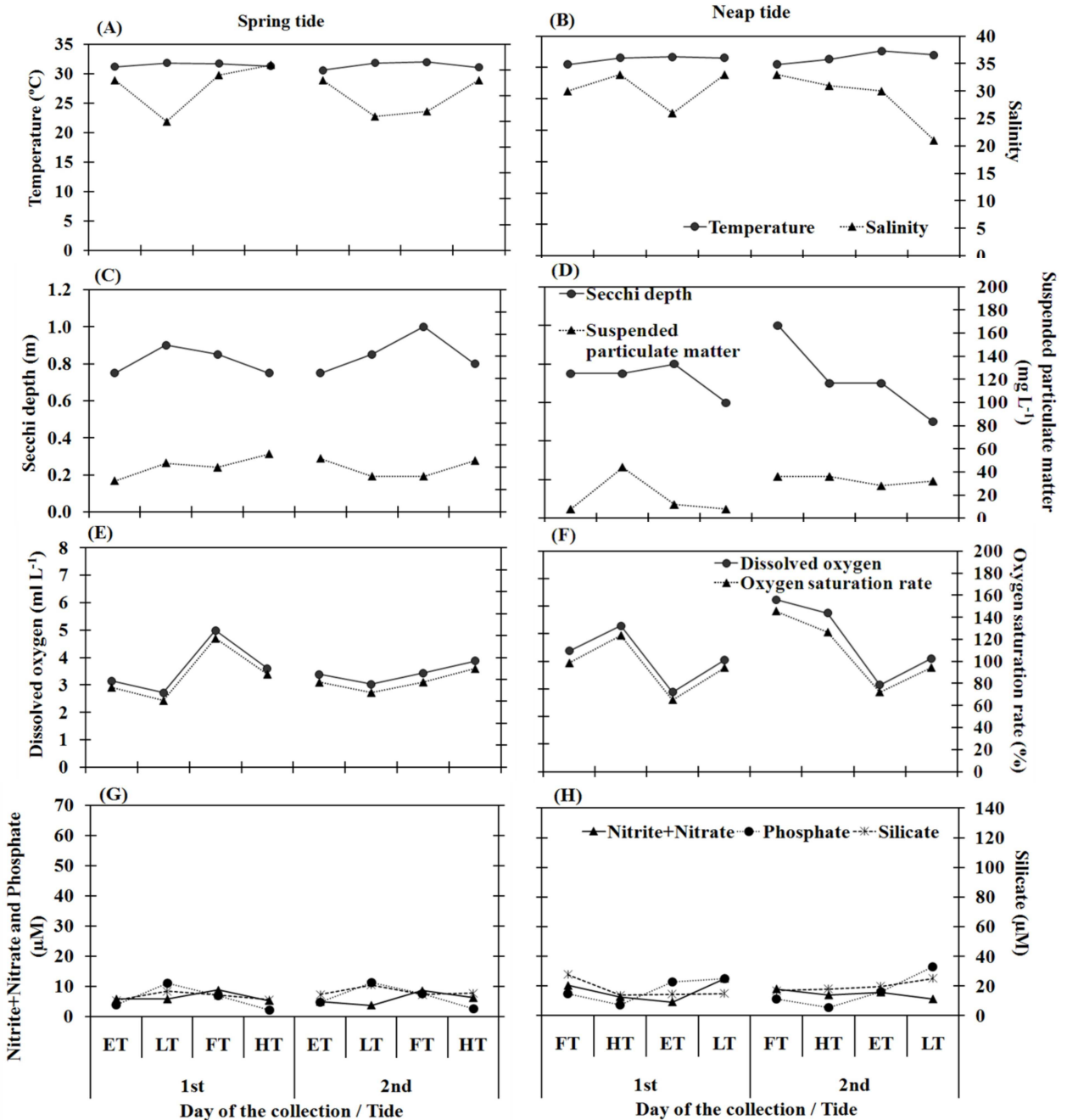

Fig. 2. Temporal variation of abiotic variables in the Port Basin of Recife (Pernambuco, Brazil) during the dry season (January 25-26 and February 02-03, 2005). Note: ST = spring tide, NT = neap tide, ET = ebb tide, LT = low tide, $\mathrm{FT}=$ flood tide and $\mathrm{HT}=$ high tide. 

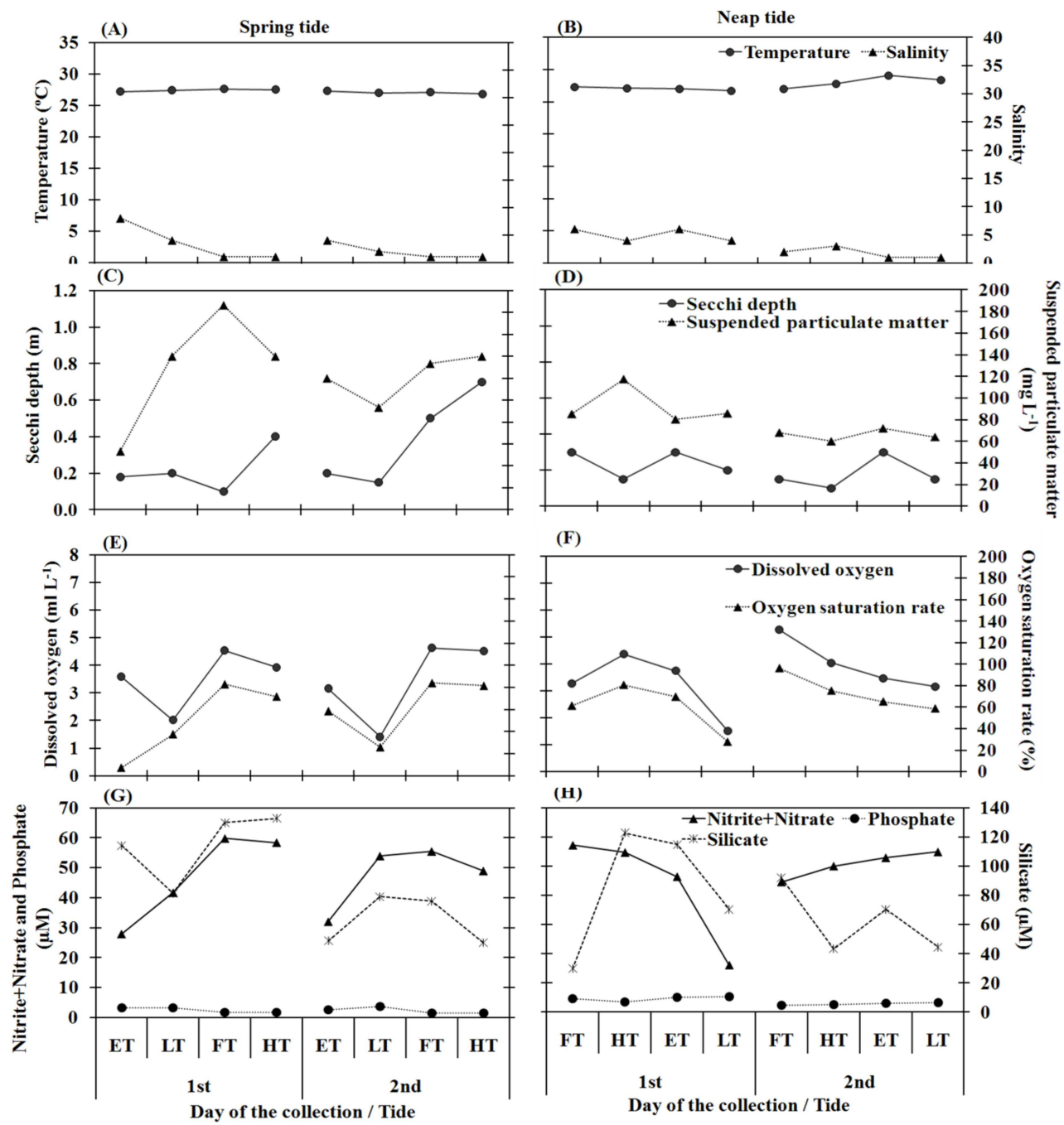

Fig. 3. Temporal variation of abiotic variables in the Port Basin of Recife Estuary (Pernambuco, Brazil) during the rainy season (June 07-08 and 14-15, 2005). Note: ST = spring tide, NT = neap tide, ET = ebb tide, $\mathrm{LT}=$ low tide, $\mathrm{FT}=$ flood tide and $\mathrm{HT}=$ high tide.

The suspended particulate material (SPM) varied from $8.0 \mathrm{mg} \mathrm{L}^{-1}$ to $187 \mathrm{mg} \mathrm{L}^{-1}$, with higher values during flood and high tides in the rainy season (Figs 2C, D; Fig. 3C, D).

No significant difference $(\mathrm{p}>0.05)$ was found between ebb tide (ET), low tide (LT), flood tide (FT) and high tide (HT) in terms of temperature, salinity, $\mathrm{pH}$, transparency and suspended particulate material. However, significant differences were found between dry and rainy seasons $(\mathrm{p}=0.000)$.

The dissolved oxygen was lower during low tide and higher during the spring and neap flood tides (Figs 2E, F; Fig. 3E, F). The minimum value was 2.72 $\mathrm{ml} \mathrm{L}^{-1}$ and the maximum was $6.24 \mathrm{ml} \mathrm{L}^{-1}$, with

significant differences between ET and LT ( $\mathrm{p}=0.006)$, ET and HT $(\mathrm{p}=0.010)$, LT and FT $(\mathrm{p}=0.001)$ and LT and HT $(\mathrm{p}=0.002)$. However, no significant differences were observed between tidal stages (ET, FT, LT, and HT) or between dry and rainy seasons ( $>0.05$ ). The dissolved oxygen saturation rate ranged from $6.93 \%$ to $145.45 \%$, with lower values in the rainy season during low tide and higher values in the dry season during flood and high tide (Figs 2E, F; Figs 3E, F). Significant differences occurred between ET and FT $(p=0.006)$, ET and HT $(p=0.013)$, LT and FT $(\mathrm{p}=0.007)$ and LT and HT $(\mathrm{p}=0.014)$ and between seasons $(\mathrm{p}=0.004)$. 
Nitrite+nitrate ranged between $3.80 \mu \mathrm{M}$ and $59.75 \mu \mathrm{M}$. Silicate ranged between $10.70 \mu \mathrm{M}$ and $133.89 \mu \mathrm{M}$, with highest concentrations during the rainy season (Figs 2G, H; Figs 3G, H). No significant differences were found among tidal stages (ET, LT, FT, HT). Significant differences occurred between dry and rainy seasons $(\mathrm{p}=0.000)$.

In general, silicate content presented a similar distribution to nitrite + nitrate, with highest values in the rainy season (Figs 2G, H; Fig. 3G, H). However, concentrations higher than $10.70 \mu \mathrm{M}$ in any season indicate that the environment has a great abundance of this nutrient. Significant differences were recorded between seasons $(p<0.05)$ but no differences were found among tidal stages.

The phosphate varied from $1.50 \mu \mathrm{M}$ to 16.40 $\mu \mathrm{M}$ and was abundant during the dry season (Figs $2 \mathrm{G}$, H; Fig. 3G, H). Significant differences were observed between ET and HT ( $p=0.035)$, LT and HT $(p=0.001)$ and between dry and rainy seasons $(\mathrm{p}=0.000)$.

\section{Phytoplankton}

Chlorophyll- $a$ concentrations varied between $3.30 \mathrm{mg} \mathrm{m}^{-3}$ and $54.40 \mathrm{mg} \mathrm{m}^{-3}$, with higher values at the low neap tide during the dry season (Figs 4A, B, $\mathrm{C}, \mathrm{D})$. There was no significant difference among tidal stages or between seasons.

A total of 127 taxa were identified: 52 in the dry season and 95 in the rainy season. In both seasons, diatoms were the group that most contributed to species richness. There were 40 diatom taxa $(76.92 \%)$ in the dry season and $58(61.05 \%)$ in the rainy season. There were 7 chlorophyte taxa $(13.46 \%)$ in the dry season and $21(22.11 \%)$ in the rainy season. Cyanobacteria were present with 4 taxa $(7.69 \%)$ in the dry season and 13 taxa $(13.68 \%)$ in the rainy season. Dinoflagellates were present only in the dry season with one taxon $(1.92 \%)$ and the euglenophytes were present only in the rainy season with three taxa $(3.16 \%)$ (Table 1).

The taxonomic richness ranged from 5 to 42 , with highest values during the rainy season (Figs $5 \mathrm{~A}$, $\mathrm{B}, \mathrm{C}, \mathrm{D})$ and significant seasonal differences $(\mathrm{p}=0.000)$.

The phytoplankton community was dominated by Helicotheca tamesis (Shrubsole) Ricard $(80.72 \%)$ during the dry season at the spring tide and by Coscinodiscus sp. $(60.87 \%)$ at neap tide (Table 2$)$. In the rainy season, Coscinodiscus centralis Ehrenberg $(53.54 \%)$ dominated in the spring tide and Oscillatoria sp. $(56.16 \%)$ in the neap tide (Table 3$)$.

Most taxa $(62.59 \%)$ occurred sporadically, followed by those of low frequency of occurrence $(28.78 \%)$, of frequent occurrence $(7.19 \%)$ and of very frequent occurrence (1.44\%). The species Oscillatoria sp. $(84.38 \%)$ and Aulacoseira granulata (Ehrenberg) Ralfs $(71.88 \%)$ were the most frequent (Table 1).

The specific diversity index ranged from

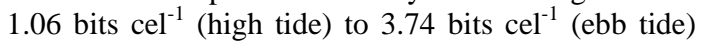
during spring tide in the dry season (Fig. 5A, B, C, D). Low species diversity was caused by the dominance of Helicotheca tamesis, Coscinodiscus centralis, Coscinodiscus kutzingii Schmidt and Aulacoseira granulate (Table 2; Table 3). There was no significant difference among tidal stages or between seasons.

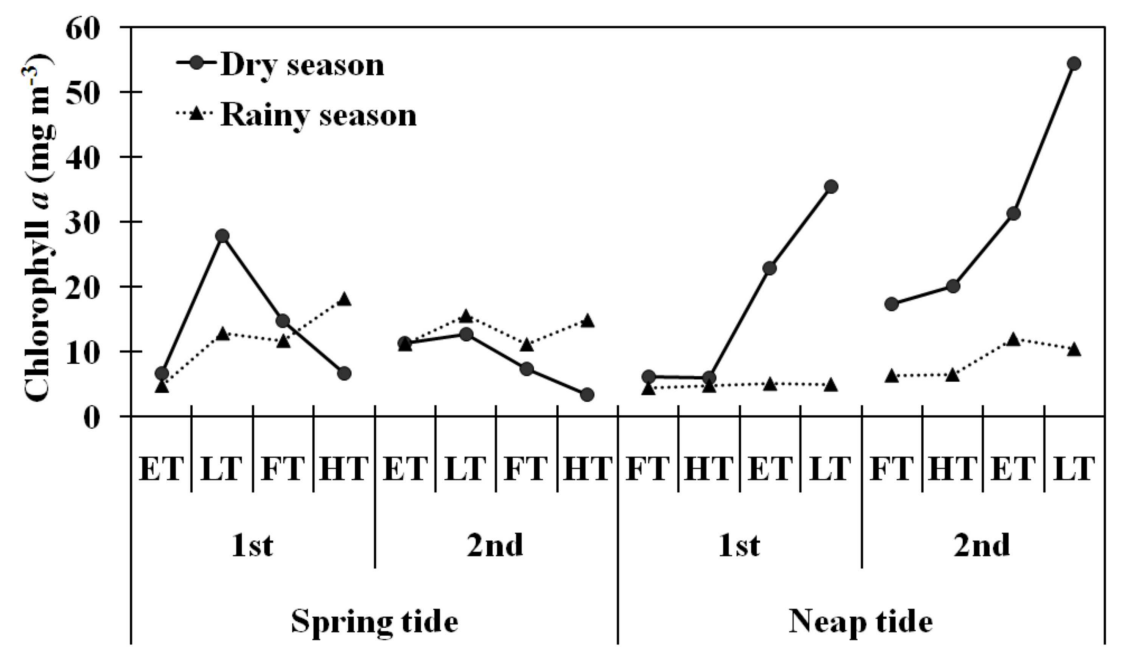

\section{Day of the collection / Tide}

Fig. 4. Temporal variation of chlorophyll- $a\left(\mathrm{mg} \mathrm{m}^{-3}\right)$ in the Port Basin of Recife Estuary (Pernambuco, Brazil) during the dry season (January 25-26 and February 02-03, 2005) and rainy season (June 07-08 and 14-15, 2005). Note: $\mathrm{ST}=$ spring tide, $\mathrm{NT}=$ neap tide, $\mathrm{ET}=$ ebb tide, $\mathrm{LT}=$ low tide, $\mathrm{FT}=$ flood tide and $\mathrm{HT}=$ high tide. 
Table 1. Microphytoplankton species checklist for the Pina Basin Estuary, Recife, Brazil, during the dry season (January 25-26 and February 02-03, 2005) and rainy season (June 07-08 and 14-15, 2005). ${ }^{* a}$ Comments: Habitat and species typical or frequent in areas: plan $=$ planktonic, tyco $=$ tycoplanktonic, $\mathrm{fw}=$ freshwater algal, est $=$ estuarine, ner $=$ neritic, oc $=$ oceanic, vfre $=$ very frequent species, fre $=$ frequent species, lfre $=$ low frequency species, espo $=$ sporadic species. Note: $\mathrm{ST}=$ spring tide, $\mathrm{NT}=$ neap tide, $\mathrm{ET}=$ ebb tide, $\mathrm{LT}=$ low tide, $\mathrm{FT}=$ flood tide and $\mathrm{HT}=$ high tide.

\begin{tabular}{|c|c|c|c|c|c|}
\hline \multirow{3}{*}{ TAXA } & \multicolumn{4}{|c|}{ DROUGHT PERIOD } & \multirow{3}{*}{ OBSERVATIONS $*$ * } \\
\hline & \multicolumn{2}{|c|}{ DRY SEASON } & \multicolumn{2}{|c|}{ RAINY SEASON } & \\
\hline & ST & NT & ST & NT & \\
\hline \multicolumn{6}{|l|}{ CYANOPHYTA } \\
\hline Anabaena sp. & ET & & ET, LT, FT, HT & FT, HT, ET, LT & lfre \\
\hline Chrooccocus dispersus (Keissler) Lemmermann & & & HT & FT, HT, ET & plan, fw, vfre \\
\hline Chrooccocus minor (Kützing) Naegeli & & & & LT & tyco, fw, spo \\
\hline Chrooccocus sp. & & & & LT & spo \\
\hline Dactylococcosis acicularis Lemmermann & & & & ET & plan, fw, spo \\
\hline Geitlerinema sp. & & & ET, LT, FT & LT, FT, HT & vfre \\
\hline Lyngbya versicolor (Wartmann) Gomont & & & ET, LT, FT & FT, HT, ET, LT & tyco, fw, spo \\
\hline Lyngbya sp. & & & FT & $\mathrm{LT}$ & fre \\
\hline Merismopedia tenuissima Lemmermann & & & & LT & tyco, fw, spo \\
\hline Oscillatoria princeps Vaucher & ET, LT & & & & tyco, fw, spo \\
\hline Oscillatoria tenuis Agardh & ET & & ET, LT, FT & FT, HT, ET, LT & tyco, fw, spo \\
\hline Oscillatoria sp. & ET, LT, FT, HT & FT, HT, ET & ET, LT, FT & FT, HT, ET, LT & vfre \\
\hline Phormidium sp. & & & & ET & spo \\
\hline \multirow{2}{*}{\multicolumn{6}{|c|}{ EUGLENOPHYTA }} \\
\hline & & & & & \\
\hline Euglena acus Ehrenberg & & & ET & LT & plan, fw, spo \\
\hline Euglena spirogyra Ehrenberg & & & & LT & plan, fw, spo \\
\hline \multirow{2}{*}{\multicolumn{6}{|c|}{$\begin{array}{l}\text { Euglena sp. } \\
\text { DINOPHYTA }\end{array}$}} \\
\hline & & & & & \\
\hline Ceratium sp. & HT & FT, HT & & & spo \\
\hline \multicolumn{6}{|l|}{ BACILLARIOPHYTA } \\
\hline Actinocyclus sp. & ET, LT, EM & & & & spo \\
\hline Amphiprora paludosa Smith & & & ET & & tyco, ner, spo \\
\hline Amphiprora sp. & & & ET & & spo \\
\hline Anomoeoneis serians (Brébisson) Cleve & & & ET & & tyco, fw, spo \\
\hline Asterionellopsis glacialis (Castracane) Round & & HT & & & tyco, ner, spo \\
\hline Aulacoseira granulata (Ehrenberg) Ralfs & ET, LT, FT & HT, LT & ET, LT, FT, HT & FT, HT, ET, LT & plan, fw, vfre \\
\hline Auliscus sp. & ET & FT, HT, ET, LT & & & lfre \\
\hline Biddulphia biddulphiana Smith & ET & $\mathrm{ET}$ & & & tyco, ner, spo \\
\hline Biddulphia tridens Ehrenberg & & & & $\mathrm{LT}$ & tyco, ner, spo \\
\hline Biddulphia sp. & ET, LT, FT, HT & FT, HT, ET, LT & & LT & lfre \\
\hline Campylodiscus clypeus Ehrenberg & & & LT, FT & & tyco, ner, spo \\
\hline Campylodiscus sp. & & & ET & & spo \\
\hline Cerataulus turgidus Ehrenberg & FT, HT & & & FT & tyco, ner, spo \\
\hline Cerataulus sp. & FT & ET & & & spo \\
\hline Chaetoceros coarctatus Lauder & & HT & & & plan, oc, spo \\
\hline Chaetoceros sp. & ET, LT, FT, HT & FT, HT, LT & & & lfre \\
\hline Climacosphenia elongata Bailey & ET & & & & tyco, ner, spo \\
\hline Coscinodiscus centralis Ehrenberg & & & ET, LT, FT, HT & ET, FT & plan, oc, lfre \\
\hline Coscinodiscus curvatulus Grunow & & LT & & & plan, oc, spo \\
\hline Coscinodiscus kutzingii Schmidt & & & ET, LT, FT, HT & FT, HT, ET, LT & plan, oc, lfre \\
\hline Coscinodiscus sp. & ET, LT, FT, HT & FT, HT, ET, LT & ET, FT & FT, HT, LT & fre \\
\hline Dimerogramma dubium Grunow & & & ET, FT & & tyco, ner, spo \\
\hline Diploneis smithii (Brébisson) Cleve & & & FT & & tyco, ner, spo \\
\hline Epithemia sp. & LT & FT & & & spo \\
\hline Eunotia sp. & & & & ET & spo \\
\hline Fragilaria capucina (Desmazière) Kützing & & & LT & & tyco, fw, spo \\
\hline Fragillaria sp. & & & ET & & spo \\
\hline Grammatophora sp. & & & ET & LT & spo \\
\hline Gyrosigma balticum (Ehrenberg) Cleve & ET, LT & FT, HT, ET, LT & ET, LT & $\mathrm{LT}$ & plan, est, lfre \\
\hline Gyrosigma strigilis (Smith) Griffith \& Henfrey & ET & & & & spo \\
\hline Helicotheca tamensis (Shrubsole) Ricard & ET, LT, FT, HT & FT, HT, ET, LT & & & plan, ner, lfre \\
\hline Hyalodiscus sp. & & & ET, LT, FT, HT & HT, ET, LT & lfre \\
\hline Licmophora flabellata (Carmichael) Agardh & HT & LT & & & tyco, ner, spo \\
\hline Licmophora sp. & LT, FT, HT & HT, ET, FT & & & lfre \\
\hline Lyrella lyra (Ehrenberg) Karayeva & ET, LT, FT & $\mathrm{LT}$ & & & tyco, ner, lfre \\
\hline
\end{tabular}




\begin{tabular}{|c|c|c|c|c|c|}
\hline \multirow{3}{*}{ TAXA } & \multicolumn{4}{|c|}{ DROUGHT PERIOD } & \multirow{3}{*}{ OBSERVATIONS ${ }^{* a}$} \\
\hline & \multicolumn{2}{|c|}{ DRY SEASON } & \multicolumn{2}{|c|}{ RAINY SEASON } & \\
\hline & ST & NT & ST & NT & \\
\hline \multicolumn{6}{|l|}{ CYANOPHYTA } \\
\hline Melosira sp. & & & ET, LT & HT, LT & lfre \\
\hline Navicula gracilis Ehrenberg & & & LT & & tyco, ner, esp \\
\hline Navicula marina Ralfs & & & & LT & tyco, ner, spo \\
\hline Navicula spp & HT & ET & ET, LT, FT, HT & FT, HT, ET, LT & spo \\
\hline Nitzschia angularis Smith & & & ET, FT, HT & ET, LT & tyco, est, lfre \\
\hline Nitzschia constricta (Kützing) Ralf & & & & LT & tyco, ner, spo \\
\hline Nitzschia distans Gregory & & & FT & & plan, oc, spo \\
\hline Nitzschia fasciculata Grunow & & & ET, LT, FT, HT & & est, lfre \\
\hline Nitzschia insignis Gregory & & & FT & & tyco, est, spo \\
\hline Nitzschia linearis (Agardh) Smith & & & ET & & tyco, fw, spo \\
\hline Nitzschia longissima (Brébisson) Grunow & & LT & & ET & tyco, fw, spo \\
\hline Nitzschia lorenziana Grunow & & & ET, LT, FT & & tyco, fw, spo \\
\hline Nitzschia máxima Grunow & & & FT & & est, spo \\
\hline Nitzschia sigma (Kützing) Smith & & & ET, LT, FT, HT & & tyco, fw, lfre \\
\hline Nitzschia sigmoidea $($ Nitzch) Smith & & & ET, LT, FT, HT & HT, LT & plan, oc, lfre \\
\hline Nitzschia thermalis (Ehrenberg) Auerswald & & & & ET & spo \\
\hline Nitzschia vermicularis (Kützing) Hantsch & & & LT, FT, HT & ET, LT & ner, lfre \\
\hline Nitzschia vitrea Norman & & & FT & & fw, spo \\
\hline Nitzschia sp. & & FT, ET & ET, LT, FT, HT & FT, HT, ET, LT & spo \\
\hline Odontella aurita (Lyngbye) Agardh & ET & & & & tyco, ner, spo \\
\hline Odontella regia (Shulltz.) Hendey & ET, FT & & & & tyco, ner, spo \\
\hline Petrodictyon gemma (Ehrenberg) Mann & ET, LT & & ET, LT, FT, HT & LT & tyco, ner, lfre \\
\hline Pinnularia major (Kützing) Ehrenberg & & & LT, FT & & tyco, ner, spo \\
\hline Pinnularia tabellaria Ehrenberg & & & LT & & tyco, ner, spo \\
\hline Plagiograma sp. & ET, LT, FT, HT & HT & & & lfre \\
\hline Pleurosigma formusum Smith & ET & & & & tyco, est, spo \\
\hline Pleurosigma speciosum Smith & & & ET, FT & & tyco, ner, spo \\
\hline Pleurosigma sp. & ET, LT, FT, HT & FT, HT, ET, LT & ET & HT, LT & fre \\
\hline Pleurosira laevis (Ehrenberg) Campère & LT & $\mathrm{LT}$ & ET, LT, FT, HT & LT & tyco, ner, lfre \\
\hline Podocystis adriatica Kützing & & & & ET & tyco, ner, spo \\
\hline Psammodictyon panduriforme (Gregory) Mann & & & ET, FT, HT & & tyco, ner, spo \\
\hline Rhabdonema sp. & & & & & spo \\
\hline Rhizosolenia styliformis Brightwell & & & HT & FT & plan, oc, spo \\
\hline Rhizosolenia sp. & ET & LT & & & spo \\
\hline Sellaphora laevissima (Kützing) Grunow & & & HT & & tyco, oc, spo \\
\hline Skeletonema costatum (Greville) Cleve & LT, FT & & & & plan, ner, spo \\
\hline Surirella spiralis Kützing & & & FT & & tyco, es, spo \\
\hline Surirella striatula Jurpin & & & FT & & tyco, es, spo \\
\hline Surirella sp. & LT, FT, HT & FT, HT, ET, LT & FT, HT & FT, HT, ET, LT & spo \\
\hline Synedra gaillonii (Bory) Ehrenberg & ET & & & & tyco, est, spo \\
\hline Synedra ulna (Nitzsch) Ehrenberg & & LT & & & tyco, fw, spo \\
\hline Synedra sp. & HT & & FT & & spo \\
\hline Tabellaria flocculosa (Roth) Kützing & LT & & & & tyco, fw, spo \\
\hline Terpsinoe americana Bailey & & & LT & & tyco, est, spo \\
\hline Terpsinoe musica Ehrenberg & & & ET, LT & HT & tyco, est, spo \\
\hline Thalassiosira eccentrica (Ehrenberg) Cleve & ET & & & LT & plan, oc, esp \\
\hline Thalassiosira sp. & ET, LT, FT & FT, HT, ET, LT & & & fre \\
\hline Triceratium alternans Bailey & & & & LT & tyco, ner, spo \\
\hline Triceratium antedeluvianum (Ehrenberg) Grunow & & & ET & & tyco, ner, spo \\
\hline Triceratium pentacrinus (Ehrenberg) Wallicia & ET & LT & & & tyco, ner, spo \\
\hline CHLOROPHYTA & & & & & \\
\hline Actinastrum hantzschii Lagerheim & & & & FT, HT, ET & plan, fw, spo \\
\hline Ankistrodesmus falcatus (Corda) Ralfs Wille & & & LT & FT, ET, LT & plan, fw, lfre \\
\hline Ankistrodesmus longissimus Lemmerman & & & ET, LT, FT & FT, HT, ET, LT & plan, fw, lfre \\
\hline Closterium dianae Ehrenberg & & & & ET & plan, fw, spo \\
\hline Closterium rostratum Ehrenberg & & & ET & HT, LT & plan, fw, spo \\
\hline Closterium setaceum Ehrenberg & & & & FT & plan, fw, spo \\
\hline Closterium striolatum Ehrenberg & & & & FT & plan, fw, spo \\
\hline Closterium sp. & & ET & & LT & spo \\
\hline Coelastrum microporum Naegeli & & LT & & & tyco, fw, spo \\
\hline Microspora sp. & ET & & & & spo \\
\hline Onychonema sp. & & & & & spo \\
\hline
\end{tabular}




\begin{tabular}{|c|c|c|c|c|c|}
\hline \multirow{3}{*}{ TAXA } & \multicolumn{4}{|c|}{ DROUGHT PERIOD } & \multirow{3}{*}{ OBSERVATIONS ${ }^{* a}$} \\
\hline & \multicolumn{2}{|c|}{ DRY SEASON } & \multicolumn{2}{|c|}{ RAINY SEASON } & \\
\hline & ST & NT & ST & NT & \\
\hline \multicolumn{6}{|l|}{ CYANOPHYTA } \\
\hline Pediastrum biwae Negoro & & & ET, LT & FT, HT, ET, LT & pfre \\
\hline Pediastrum duplex Meyen & FT & & ET, LT, FT, HT & FT, HT, ET, LT & plan, fw, spo \\
\hline Pediastrum simplex Meyen & & & & FT, ET & plan, fw, spo \\
\hline Pediastrum sp. & & & FT & $\mathrm{LT}$ & spo \\
\hline Scenedesmus quadricauda var. longispina Chod & & & LT, FT, HT & FT, HT, ET, LT & plan, fw, lfre \\
\hline Scenedesmus sp. & & LT & ET & & spo \\
\hline Spondylosium $\mathrm{sp}$ & & & & ET, LT & spo \\
\hline Staurastrum gracile Ralfs & ET, FT & & ET, LT & HT, LT & tyco, fw, lfre \\
\hline Staurastrum leptocladum Nordstedt & & & ET, LT, FT, HT & FT, HT, ET, LT & tyco, fw, lfre \\
\hline Staurastrum longiradiatum West \& West & & & LT & & tyco, fw, lfre \\
\hline Staurastrum subanchora Gronbl. & & & ET & & tyco, fw, lfre \\
\hline Staurastrum subindentatum (West \& West) Teiling & & & LT & & tyco, fw, lfre \\
\hline Staurastrum sp. & ET, HT & HT & ET, LT, FT & FT, LT & lfre \\
\hline
\end{tabular}

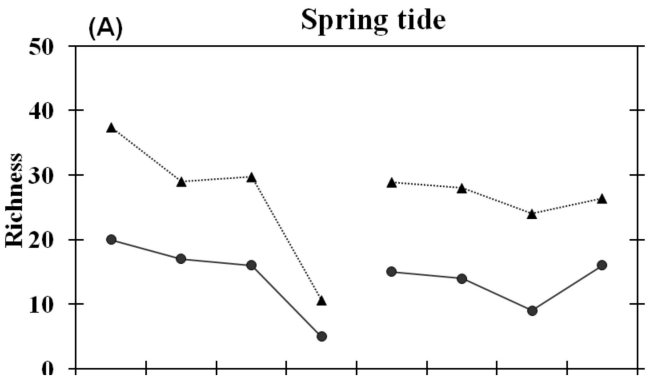

(C)

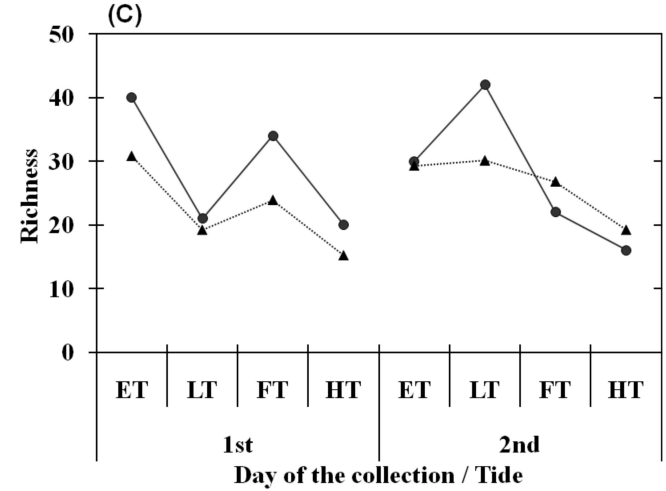

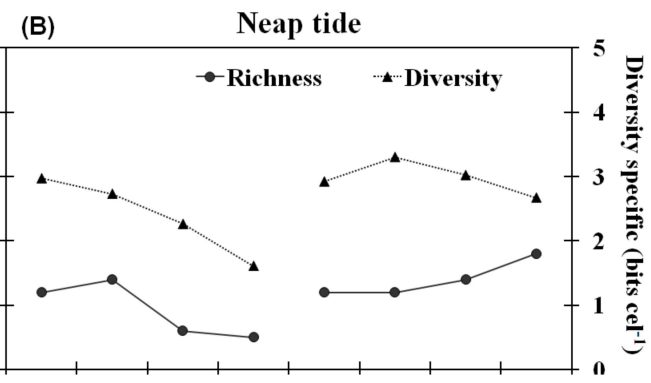

(D)

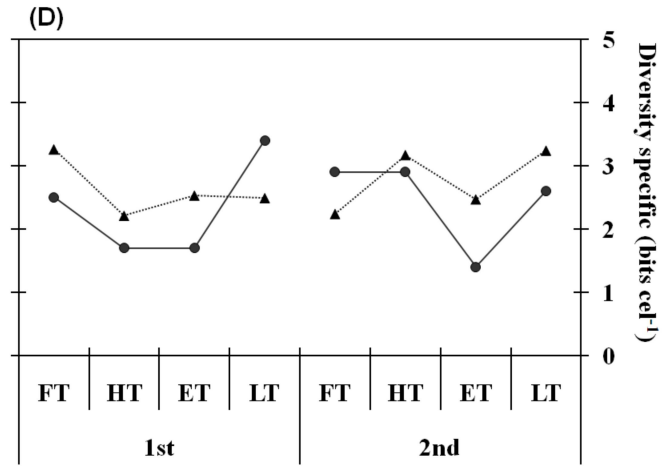

Day of the collection / Tide

Fig. 5. Temporal variation of biotic variables in the Port Basin of Recife Estuary (Pernambuco, Brazil) during the dry season (January 25-26 and February 02-03, 2005) (A, B) and rainy season (June 07-08 and 14-15, 2005) (C, D). Note: ST = spring tide, $\mathrm{NT}=$ neap tide, $\mathrm{ET}=$ ebb tide, $\mathrm{LT}=$ low tide, $\mathrm{FT}=$ flood tide and $\mathrm{HT}=$ high tide .

Table 2. Relative abundance (\%) of the microphytoplankton species in the Pina Basin Estuary, Recife, Brazil, during the dry season (January 25-26 and February 02-03, 2005). Note: ST = spring tide, NT = neap tide, $\mathrm{ET}=$ ebb tide, $\mathrm{LT}=$ low tide, FT = flood tide and $\mathrm{HT}=$ high tide.

\begin{tabular}{|c|c|c|c|c|c|c|c|c|c|c|c|c|c|c|c|c|}
\hline & \multicolumn{16}{|c|}{ DROUGHT PERIOD } \\
\hline & \multicolumn{8}{|c|}{ SPRING TIDE } & \multicolumn{8}{|c|}{ NEAP TIDE } \\
\hline & \multicolumn{4}{|c|}{$1^{\text {st }}$} & \multicolumn{4}{|c|}{$2^{\text {nd }}$} & \multicolumn{4}{|c|}{$1^{\text {st }}$} & \multicolumn{4}{|c|}{$2^{\text {nd }}$} \\
\hline & ET & LT & FT & HT & ET & LT & FT & HT & FT & HT & ET & LT & FT & HT & ET & LT \\
\hline \multicolumn{17}{|l|}{ CYANOPHYTA } \\
\hline $\begin{array}{l}\text { Anabaena sp. } \\
\text { Oscillatoria princeps } \\
\text { Vaucher }\end{array}$ & $\begin{array}{l}1.69 \\
1.69\end{array}$ & 0.74 & & & & & & & & & & & & & & \\
\hline
\end{tabular}


Table 2. Continuation.

\begin{tabular}{|c|c|c|c|c|c|c|c|c|c|c|c|c|c|c|c|c|}
\hline & \multicolumn{16}{|c|}{ DROUGHT PERIOD } \\
\hline & \multicolumn{8}{|c|}{ SPRING TIDE } & \multicolumn{8}{|c|}{ NEAP TIDE } \\
\hline & \multicolumn{4}{|c|}{$1^{\text {st }}$} & \multicolumn{4}{|c|}{$2^{\text {nd }}$} & \multicolumn{4}{|c|}{$1^{\text {st }}$} & \multicolumn{4}{|c|}{$2^{\text {nd }}$} \\
\hline & ET & LT & FT & HT & ET & LT & FT & HT & FT & HT & ET & LT & FT & HT & ET & LT \\
\hline Oscillatoria tenuis Agardh & 3.39 & & & & & & & & & & & & & & & \\
\hline $\begin{array}{l}\text { Oscillatoria sp. } \\
\text { DINOPHYTA }\end{array}$ & 27.12 & 20.74 & 13.66 & 6.02 & 4.14 & 21.03 & 5.88 & 5.19 & 16.67 & & 11.11 & 5.00 & 4.17 & & 32.61 & 15.00 \\
\hline $\begin{array}{l}\text { Ceratium sp. } \\
\text { BACILLARIOPHYTA }\end{array}$ & & & & & & & & 1.30 & & & 1.59 & 0.83 & & & & \\
\hline Actinocyclus sp. & 5.08 & 1.48 & 2.73 & & & & & & & & & & & & & \\
\hline $\begin{array}{l}\text { Asterionellopsis glacialis } \\
\text { (Castr.) Round }\end{array}$ & & & & & & & & & & & & 0.83 & & & & \\
\hline $\begin{array}{l}\text { Aulacoseira granulata (Ehr.) } \\
\text { Ralfs }\end{array}$ & 8.47 & 5.93 & 2.73 & & 5.52 & 5.13 & & & & & & 0.83 & & 0.87 & & 5.00 \\
\hline Auliscus sp. & 1.69 & & & & & & & & 5.56 & & & & 2.08 & 0.43 & 4.35 & 5.00 \\
\hline $\begin{array}{l}\text { Biddulphia biddulphiana } \\
\text { Smith }\end{array}$ & & & & & 0.69 & & & & & & & & 3.13 & & & \\
\hline Biddulphia sp. & & 0.74 & 4.37 & & 2.76 & 0.51 & & 5.19 & & 4.35 & 1.59 & & 16.67 & & 6.52 & 5.00 \\
\hline Cerataulus turgidus Ehrenberg & & & 8.20 & 7.23 & & & & & & & & & & & & \\
\hline Cerataulus sp. & & & & & & & 7.84 & & 11.11 & & & & & & & \\
\hline Chaetoceros coarctatus Laud. & & & & & & & & & & & & 20.83 & & & & 5.00 \\
\hline Chaetoceros sp. & & 2.22 & 0.55 & & 1.38 & 2.05 & 2.61 & 5.19 & & & 4.76 & 35.83 & & 6.49 & 6.52 & 5.00 \\
\hline Climacosphenia elongata Bail. & 1.69 & & & & & & & & & & & & & & & \\
\hline Coscinodiscophyceae & 3.39 & & & & 4.83 & 3.08 & & 6.49 & & & & & 12.50 & 1.30 & 2.17 & 10.00 \\
\hline $\begin{array}{l}\text { Coscinodiscus curvatulus } \\
\text { Grun. }\end{array}$ & & & & & & & & & & & & & & 3.46 & & \\
\hline Coscinodiscus sp. & & 10.37 & 2.73 & 4.82 & 12.41 & 15.38 & 22.88 & 9.09 & 22.22 & 60.87 & 17.46 & 10.83 & 31.25 & 17.75 & 23.91 & 10.00 \\
\hline Epithemia sp. & & 0.74 & & & & & & & & & 6.35 & & & & & \\
\hline Gyrosigma balticum (Ehr.) Cl. & 10.17 & 1.48 & & & & & & & & & 3.17 & 2.50 & 1.04 & 1.30 & & \\
\hline Gyrosigma strigilis (Sm.) Grif. & 6.78 & & & & 0.69 & & & & & & & & & & & \\
\hline $\begin{array}{l}\text { Helicotheca thamensis (Shrub.) } \\
\text { Ricard }\end{array}$ & & & 32.24 & 80.72 & 42.76 & 21.54 & 35.29 & 53.25 & & 8.70 & 4.76 & 15.00 & 2.08 & & 6.52 & 25.00 \\
\hline $\begin{array}{l}\text { Licmophora flabellata (Carm.) } \\
\text { Agardh }\end{array}$ & & & & & & & & 1.30 & & & & & & 0.87 & & \\
\hline Licmophora sp. & & 0.74 & 0.55 & 1.20 & & 0.51 & & 1.30 & 5.56 & & 1.59 & 0.83 & & & 2.17 & \\
\hline Lyrella lyra (Ehr.) Karayeva & 3.39 & 3.70 & 0.55 & & & & & 1.30 & & & & & & 0.43 & & \\
\hline Navicula sp1 & & & & & & & & 1.30 & & & & & 1.04 & & & \\
\hline $\begin{array}{l}\text { Nitzschia longissima (Bréb.) } \\
\text { Grunow }\end{array}$ & & & & & & & & & & & & & & 41.13 & & \\
\hline Nitzschia sp. & & & & & & & & & & & & & 1.04 & & 6.52 & \\
\hline Odontella aurita (Lyngb.) Ag. & 6.78 & & & & & & & & & & & & & & & \\
\hline Odontella regia (Shulttz.) Hen. & 3.39 & & 0.55 & & & & & & & & & & & & & \\
\hline $\begin{array}{l}\text { Petrodictyon gemma (Ehr.) } \\
\text { Mann }\end{array}$ & & & & & 0.69 & 0.51 & & & & & & & & & & \\
\hline Plagiograma sp. & & 2.22 & 2.19 & & 5.52 & 0.51 & 1.96 & 1.30 & & & & 0.83 & & & & \\
\hline Pleurosigma formusum Smith & 3.39 & & & & & & & & & & & & & & & \\
\hline Pleurosigma sp. & & & 4.92 & & 9.66 & 5.13 & 0.65 & 3.90 & & & 19.05 & 1.67 & 3.13 & 1.73 & 2.17 & \\
\hline Pleurosira laevis (Ehr.) Camp. & & 8.89 & & & & & & & & & & & & 3.90 & & \\
\hline Rhizosolenia sp. & & & & & 0.69 & & & & & & & & & 0.43 & & \\
\hline $\begin{array}{l}\text { Skeletonema costatum } \\
\text { (Greville) Cleve }\end{array}$ & & 1.48 & 2.19 & & & & & & & & & & & & & \\
\hline Surirella sp. & & 0.74 & & & & 0.51 & 1.31 & 1.30 & & 4.35 & 1.59 & 1.67 & 11.46 & & 4.35 & \\
\hline Synedra gaillonii (Bory) Ehr. & 1.69 & & & & & & & & & & & & & & & \\
\hline Synedra ulna (Nitzsch) Ehr. & & & & & & & & & & & & & & 0.43 & & \\
\hline Synedra sp. & & & & & & & & 1.30 & & & & & & & & \\
\hline
\end{tabular}


Table 2. Continuation.

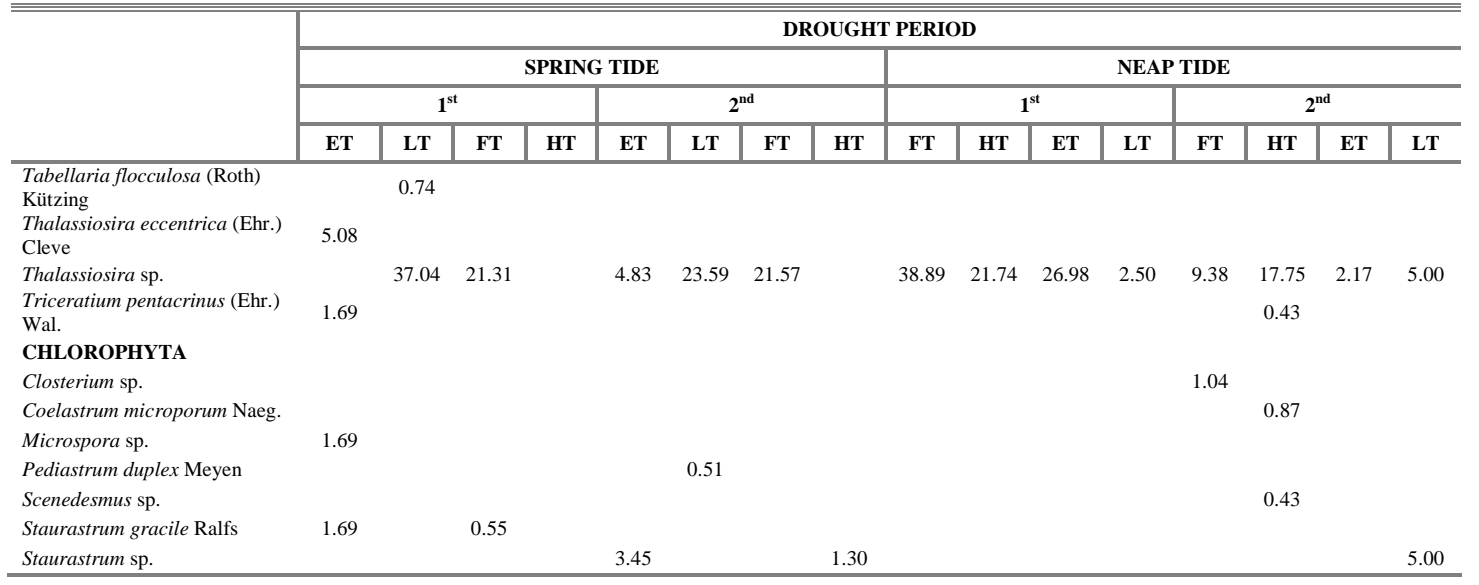

Table 3. Relative abundance $(\%)$ of microphytoplankton species in the Pina Basin Estuary, Recife, Brazil, during the rainy season (June 07-08 and 14-15, 2005). Note: ST = spring tide, NT = neap tide, ET = ebb tide, $\mathrm{LT}=$ low tide, FT = flood tide and $\mathrm{HT}=$ high tide.

\begin{tabular}{|c|c|c|c|c|c|c|c|c|c|c|c|c|c|c|c|c|}
\hline & \multicolumn{16}{|c|}{ DROUGHT PERIOD } \\
\hline & \multicolumn{8}{|c|}{ SPRING TIDE } & \multicolumn{8}{|c|}{ NEAP TIDE } \\
\hline & \multicolumn{4}{|c|}{$1^{\text {st }}$} & \multicolumn{4}{|c|}{$2^{\text {nd }}$} & \multicolumn{4}{|c|}{$1^{\text {st }}$} & \multicolumn{4}{|c|}{$2^{\text {nd }}$} \\
\hline & ET & LT & FT & HT & ET & LT & FT & HT & FT & HT & ET & LT & FT & HT & ET & LT \\
\hline \multicolumn{17}{|l|}{ CYANOPHYTA } \\
\hline Anabaena sp. & 0.98 & & 4.68 & & 0.30 & 0.31 & & 0.19 & & 0.72 & 0.25 & & 2.53 & & & 4.32 \\
\hline $\begin{array}{l}\text { Chrooccocus dispersus } \\
\text { (Keissler) Lemmermann } \\
\text { Chrooccocus minor (Kützing) } \\
\text { Naegeli }\end{array}$ & & & & & & & & 0.19 & & 0.48 & & 0.38 & 0.37 & & 0.20 & 0.38 \\
\hline Chrooccocus sp. & & & & & & & & & & & & & & 0.21 & & \\
\hline Cyanophyceae & 15.20 & & 3.71 & & 8.73 & 5.98 & & 0.56 & 21.96 & 17.12 & 11.68 & 47.71 & 0.95 & 2.12 & 50.84 & 34.77 \\
\hline $\begin{array}{l}\text { Dactylococcosis acicularis } \\
\text { Lemmermann }\end{array}$ & & 7.60 & & & & & & & & & & & 0.11 & & & \\
\hline Geitlerinema sp. & 1.47 & & 0.52 & & 0.53 & & & & & 0.56 & 15.45 & 2.86 & & & 0.39 & 1.32 \\
\hline $\begin{array}{l}\text { Lyngbya versicolar (Wartmann) } \\
\text { Gomont }\end{array}$ & & & & & & & 0.14 & & & & & & & 0.11 & & \\
\hline Lyngbya sp. & 0.34 & & 0.13 & & 0.08 & 0.58 & 0.36 & & 3.38 & 0.80 & 3.39 & 0.95 & 0.11 & 0.32 & 0.69 & 0.19 \\
\hline $\begin{array}{l}\text { Merismopedia tenuissima } \\
\text { Lemmermann }\end{array}$ & & & & & & & & & & 0.88 & & & & & & \\
\hline Oscillatoria sp. & 5.74 & 21.77 & 2.86 & & 9.86 & 27.48 & 4.84 & & 21.96 & 56.16 & 25.88 & 30.92 & 49.47 & 27.60 & 10.56 & 13.72 \\
\hline Oscillatoriaceae & 0.34 & & & & & & 10.33 & & 37.16 & & 0.38 & & 2.32 & 11.04 & & \\
\hline Phormidium sp. & & & & & & & & & 0.34 & & & & & & & \\
\hline $\begin{array}{l}\text { Planktolyngbya microspira } \\
\text { Komarek }\end{array}$ & 1.37 & 1.44 & 0.55 & & 1.51 & 0.23 & 1.00 & & & 0.40 & 1.51 & 1.72 & 3.11 & & 0.99 & 3.95 \\
\hline \multicolumn{17}{|l|}{ EUGLENOPHYTA } \\
\hline Euglena acus Ehrenberg & & & & & 0.08 & & & & & 0.24 & & & & & & \\
\hline Euglena spirogyra Ehrenberg & & & & & & & & & & 0.08 & & & & & & \\
\hline Euglena sp. & & & & & & & & & & & & & & 0.11 & & \\
\hline \multicolumn{17}{|l|}{ BACILLARIOPHYTA } \\
\hline Amphiprora paludosa Smith & & 0.21 & & & 0.08 & & & & & & & & & & & \\
\hline Amphiprora sp. & 0.05 & & & & & & & & & & & & & & & \\
\hline Anomoeoneis serians (Bréb.) Cl. & 0.05 & & & & & & & & & & & & & & & \\
\hline $\begin{array}{l}\text { Aulacoseira granulata } \\
\text { (Ehrenberg) Ralfs }\end{array}$ & 27.66 & & 47.23 & 37.60 & 35.09 & 22.67 & 39.89 & 37.01 & 2.03 & 6.00 & 17.09 & 4.58 & 16.32 & 19.96 & 17.97 & 9.96 \\
\hline Bacillariophyceae & 6.52 & 0.62 & & & 2.64 & 1.28 & 0.21 & 1.31 & & 3.12 & & 1.34 & & 0.21 & & 2.44 \\
\hline Biddulphia tridens Ehrenberg & & & & & & & & & & & & & & 0.11 & & \\
\hline
\end{tabular}


Table 3. Continuation.

\begin{tabular}{|c|c|c|c|c|c|c|c|c|c|c|c|c|c|c|c|c|}
\hline & \multicolumn{16}{|c|}{ DROUGHT PERIOD } \\
\hline & \multicolumn{8}{|c|}{ SPRING TIDE } & \multicolumn{8}{|c|}{ NEAP TIDE } \\
\hline & \multicolumn{4}{|c|}{$1^{\text {st }}$} & \multicolumn{4}{|c|}{$2^{\text {nd }}$} & \multicolumn{4}{|c|}{$1^{\text {st }}$} & \multicolumn{4}{|c|}{$2^{\text {nd }}$} \\
\hline & ET & LT & FT & HT & ET & LT & FT & HT & FT & HT & ET & LT & FT & HT & ET & LT \\
\hline Biddulphia sp. & & & & & & & & & & 0.16 & & & & & & \\
\hline Campylodiscus clypeus Ehr. & & & 0.10 & & & 0.27 & & & & & & & & & & \\
\hline Campylodiscus sp. & 0.05 & & & & & & & & & & & & & & & \\
\hline Cerataulus turgidus Ehrenberg & & & & & & & & & & & 0.25 & & & & & \\
\hline Cerataulus sp. & & 1.03 & & & & & & & & & & & & & & \\
\hline Coscinodiscophyceae & & & & 0.10 & & & & & & & & & & & & \\
\hline Coscinodiscus centralis Ehr. & 0.05 & & 0.10 & 53.54 & & 0.39 & & & 3.72 & & 3.89 & & & & & \\
\hline Coscinodiscus Kutzingii Schm. & 27.12 & 15.61 & 27.81 & & 23.19 & 16.30 & 17.66 & 45.42 & & 2.72 & & 2.48 & 2.63 & 3.40 & 6.91 & 2.82 \\
\hline Coscinodiscus sp. & 0.98 & 0.21 & & & 0.53 & & 1.28 & & & 0.48 & 0.25 & & & 2.34 & & 2.63 \\
\hline Dimeregramma dubium Grun. & & & & & 0.08 & & 0.07 & & & & & & & & & \\
\hline Diploneis smithii (Bréb.) Cleve & & & 0.03 & & & & & & & & & & & & & \\
\hline Eunotia sp. & & & & & & & & & 0.34 & & & & & & & \\
\hline $\begin{array}{l}\text { Fragilaria capucina (Desm.) } \\
\text { Kützing }\end{array}$ & & & & & & 0.04 & & & & & & & & & & \\
\hline Fragillaria sp. & 0.05 & & & & & & & & & & & & & & & \\
\hline Grammatophora sp. & 0.15 & & & & & & & & & 0.56 & & & & & & \\
\hline Gyrosigma balticum (Ehr.) Cl. & 0.15 & & & & & 0.08 & & & & & & & & 0.21 & & \\
\hline Hyalodiscus sp. & 1.18 & 0.21 & 0.03 & 0.10 & & 0.19 & 0.64 & & & 0.24 & & & 0.11 & & & 0.19 \\
\hline Melosira sp. & 2.16 & 35.73 & & & 1.05 & 13.59 & & & & 2.24 & & & & & & 0.19 \\
\hline Navicula gracilis Ehrenberg & & & & & & 0.04 & & & & & & & & & & \\
\hline Navicula marina Ralfs & & & & & & & & & & 0.08 & & & & & & \\
\hline Navicula sp1 & 0.29 & 0.41 & 0.20 & 0.10 & & 1.09 & 0.07 & 0.19 & 0.34 & 1.76 & & 0.19 & 0.16 & & & 0.19 \\
\hline Navicula $\mathrm{sp} 2$ & & 0.41 & 0.07 & 0.10 & & 0.08 & & & & & & & 0.05 & & & \\
\hline Nitzschia angularis Smith & 0.05 & & 0.16 & 0.21 & 0.30 & & 0.14 & 0.75 & & & & & 0.21 & 0.21 & & \\
\hline $\begin{array}{l}\text { Nitzschia constricta (Kützing) } \\
\text { Ralf }\end{array}$ & & & & & & & & & & 0.08 & & & & & & \\
\hline Nitzschia distans Gregory & & & & & & 0.08 & & & & & & & & & & \\
\hline Nitzschia fasciculata Grunow & 0.10 & & 0.46 & 0.21 & 0.75 & 0.08 & & 0.75 & & & & & & & & \\
\hline Nitzschia insignis Gregory & & & & & & 0.08 & & & & & & & & & & \\
\hline Nitzschia linearis (Ag.) Smith & & & & & 0.08 & & & & & & & & & & & \\
\hline $\begin{array}{l}\text { Nitzschia longissima (Bréb.) } \\
\text { Grunow }\end{array}$ & & & & & & & & & & & & & 0.05 & & & \\
\hline Nitzschia lorenziana Grunow & 0.29 & 0.41 & 0.13 & & & 0.31 & & & & & & & & & & \\
\hline Nitzschia maximaGrunow & & & 0.07 & & & & 0.07 & & & & & & & & & \\
\hline Nitzschia sigma (Kütz.) Smith & 0.10 & 1.03 & 0.33 & 0.31 & 0.08 & 0.08 & & & & & & & & & & \\
\hline Nitzschia sigmoidea (Nitz.) Sm & 0.54 & & 0.46 & 0.10 & 0.68 & 0.08 & & 0.37 & & 0.08 & & & & & & 0.19 \\
\hline Nitzschia thermalis (Ehr.) Aue. & & & & & & & & & & & & & 0.11 & & & \\
\hline $\begin{array}{l}\text { Nitzschia vermicularis (Kützing) } \\
\text { Hantsch }\end{array}$ & & & 0.33 & 0.10 & & 0.04 & 0.14 & & & & & & 0.16 & 0.42 & & \\
\hline Nitzschia vitrea Norman & & & 0.07 & & & & & & & & & & & & & \\
\hline Nitzschia sp. & 0.78 & 0.62 & 1.01 & 0.21 & 1.28 & 1.05 & 3.28 & 0.56 & 0.68 & 0.88 & 0.38 & & 0.26 & 0.32 & & 0.19 \\
\hline Petrodictyon gemma (Ehr.) M. & 1.91 & & 5.86 & 5.83 & 8.66 & 3.53 & & 9.72 & & 0.40 & & & & & & \\
\hline Pinnularia major (Kütz.) Ehr. & & & 0.03 & & & 0.04 & & & & & & & & & & \\
\hline Pinnularia tabellaria Ehr. & & & & & & 0.04 & & & & & & & & & & \\
\hline Pleurosigma speciosum Smith & 0.05 & & 0.03 & & & & & & & & & & & & & \\
\hline Pleurosigma sp. & 0.25 & 0.62 & & & & & & & & 0.64 & & 0.57 & & & & 0.56 \\
\hline Pleurosira laevis (Ehr.) Camp. & 0.34 & & 1.56 & 0.21 & 1.28 & 1.24 & & 0.93 & & 0.88 & & & & & & \\
\hline Podocystis adriatica Kützing & & & & & & & & & 0.34 & & & & & & & \\
\hline $\begin{array}{l}\text { Psammodictyon panduriforme } \\
\text { (Greg.) Mann }\end{array}$ & 0.05 & & 0.03 & 0.10 & & & & & & & & & & & & \\
\hline Rhabdonema sp. & & & & & & & & & 0.68 & & & & & & & \\
\hline Rhizosolenia styliformis Brigh. & & & & 0.10 & & & & & & & 0.25 & & & & & \\
\hline $\begin{array}{l}\text { Sellaphora laevissima (Kützing) } \\
\text { Grunow }\end{array}$ & & & & 0.10 & & & & & & & & & & & & \\
\hline Surirella spiralis Kützing & & & 0.03 & & & & & & & & & & & & & \\
\hline
\end{tabular}




\begin{tabular}{|c|c|c|c|c|c|c|c|c|c|c|c|c|c|c|c|c|}
\hline & \multicolumn{16}{|c|}{ DROUGHT PERIOD } \\
\hline & \multicolumn{8}{|c|}{ SPRING TIDE } & \multicolumn{8}{|c|}{ NEAP TIDE } \\
\hline & \multicolumn{4}{|c|}{$1^{\text {st }}$} & \multicolumn{4}{|c|}{$2^{\text {nd }}$} & \multicolumn{4}{|c|}{$1^{\mathrm{st}}$} & \multicolumn{4}{|c|}{$2^{\text {nd }}$} \\
\hline & ET & LT & FT & HT & ET & LT & FT & HT & FT & HT & ET & LT & FT & HT & ET & LT \\
\hline Surirella striatula Jurpin & & 6.78 & & & & & 0.78 & & & & & & & & & \\
\hline Surirella sp. & & & & & & & 4.27 & 0.19 & & & 0.25 & & 0.42 & 0.74 & & 0.19 \\
\hline Synedra sp. & & & & & & & 0.14 & & & & & & & & & \\
\hline Terpsinoe americana Bailey & & & & & & 0.04 & & & & & & & & & & \\
\hline Terpsinoe musica Ehrenberg & 0.25 & & & & & 0.12 & & & & & & 0.19 & & & & \\
\hline $\begin{array}{l}\text { Thalassiosira eccentrica } \\
\text { (Ehrenberg) Cleve }\end{array}$ & & & & & & & & & & & & & & 0.21 & & \\
\hline Triceratium alternans Bailey & & & & & & & & & & 0.64 & & & & 0.21 & & \\
\hline $\begin{array}{l}\text { Triceratium antedeluvianum } \\
\text { (Ehrenberg) Grunow }\end{array}$ & & & & & 0.08 & & & & & & & & & & & \\
\hline \multicolumn{17}{|l|}{ CHLOROPHYTA } \\
\hline Actinastrum hantzschii Lag. & & & & & & & & & & & & & 0.05 & & 0.20 & 0.38 \\
\hline $\begin{array}{l}\text { Ankistrodesmus falcatus (Corda) } \\
\text { Ralfs Wille }\end{array}$ & & & & & & 0.04 & & & 0.34 & & 0.75 & & 0.42 & 1.70 & 0.20 & \\
\hline $\begin{array}{l}\text { Ankistrodesmus longissimus } \\
\text { Lemm. }\end{array}$ & 0.93 & & 0.13 & & 0.30 & 0.04 & & & & 0.08 & 8.42 & 4.01 & 15.53 & 12.74 & 6.42 & 10.90 \\
\hline Chlorophyceae & 0.74 & 0.21 & 0.72 & & 0.90 & 0.54 & 14.32 & 1.68 & 5.07 & 0.88 & 2.51 & 1.34 & & 7.75 & 4.05 & 8.27 \\
\hline Closterium dianae Ehrenberg & & & & & & & & & & & & & 0.11 & & & \\
\hline Closterium rostratum Ehr. & & & & & 0.08 & & & & & 0.24 & & & & & & 0.38 \\
\hline Closterium setaceum Ehr. & & & & & & & & & & & 0.13 & & & & & \\
\hline Closterium striolatum Ehr. & & & & & & & & & & & 0.25 & & & & & \\
\hline Closterium sp. & & & & & & & & & 0.34 & & & & & 0.21 & & \\
\hline Onychonema sp. & & & & & & & & & 0.68 & & & & & & & \\
\hline Pediastrum biwae Negoro & 0.05 & 0.62 & & & 0.15 & 0.08 & & & & 0.08 & 2.64 & & 2.63 & 0.53 & 0.10 & 0.38 \\
\hline Pediastrum duplex Meyen & & & & 0.10 & 0.08 & 0.04 & 0.07 & 0.19 & 0.68 & & 2.76 & 0.38 & 1.32 & 2.23 & 0.49 & 1.13 \\
\hline Pediastrum simplex Meyen & & & & & & & & & & & 0.38 & & 0.11 & & & \\
\hline Pediastrum sp. & & & 0.03 & & & & & & & & & & & 0.53 & & \\
\hline Scenedesmus longispina Chod & & & 0.07 & 0.10 & & 0.04 & & & & 0.08 & 0.63 & 0.19 & 0.11 & & & 0.19 \\
\hline Scenedesmus sp. & 0.10 & & & & & & & & & & & & & & & \\
\hline Spondylosium sp. & & & & & & & & & & & & & 0.05 & 3.93 & & \\
\hline Staurastrum gracile Ralfs & 0.05 & & & & & 0.12 & & & & 0.08 & & 0.19 & & & & \\
\hline Staurastrum leptocladum Nor. & 1.47 & 4.11 & 0.49 & 0.73 & 1.43 & 1.16 & & & & 0.16 & 0.50 & & 0.26 & 0.32 & & 0.19 \\
\hline $\begin{array}{l}\text { Staurastrum longiradiatum } \\
\text { West and West }\end{array}$ & & & & & & 0.04 & & & & & & & & & & \\
\hline $\begin{array}{l}\text { Staurastrum subanchora } \\
\text { Gronbl. }\end{array}$ & 0.05 & & & & & & & & & & & & & & & \\
\hline Staurastrum subindentatum & & & & & & 0.19 & & & & & & & & & & \\
\hline Staurastrum sp. & & 0.41 & & & 0.15 & 0.31 & 0.28 & & & & 0.13 & & & 0.21 & & \\
\hline
\end{tabular}

The first two PCA components accounted for $67.59 \%$ of the total variation. The first factor accounted for $53.39 \%$ and the second for $14.20 \%$. The bi-dimensional projection shows two groups of variables with high internal correlation. Group A includes salinity, water temperature, water transparency and phosphate and characterizes the dry season. Group B brought together species richness, nitrogen (nitrate+nitrite), silicate and suspended particulate matter and characterized the rainy season. The dissolved oxygen and chlorophyll- $a$ were related to the second axis and were inversely correlated to the species diversity (Fig. 6).

From the similarity analysis of the samples it was possible to distinguish two groups of species corresponding to the dry and rainy seasons (Fig. 7) when significant differences $(p=0.001)$ were observed.
However, no significant differences were observed among the stages or between spring and neap tides.

\section{Discussion}

A multitude of physical, chemical and biological processes affect organisms in marine areas (MANN, 1982). These processes operate over a range of spatial and temporal scales (TAGUSHI; LAWS, 1987) that must be considered when explaining variability in the structure, function and distribution of phytoplankton communities. In the tropics, this variability is highly affected by seasonal changes in rainfall (SOURNIA, 1969). Seasonal rainfall in tropical areas produces temporal and regional differences in river discharge. This induces fluctuations in salinity, nutrient concentrations, turbidity and biological productivity. 


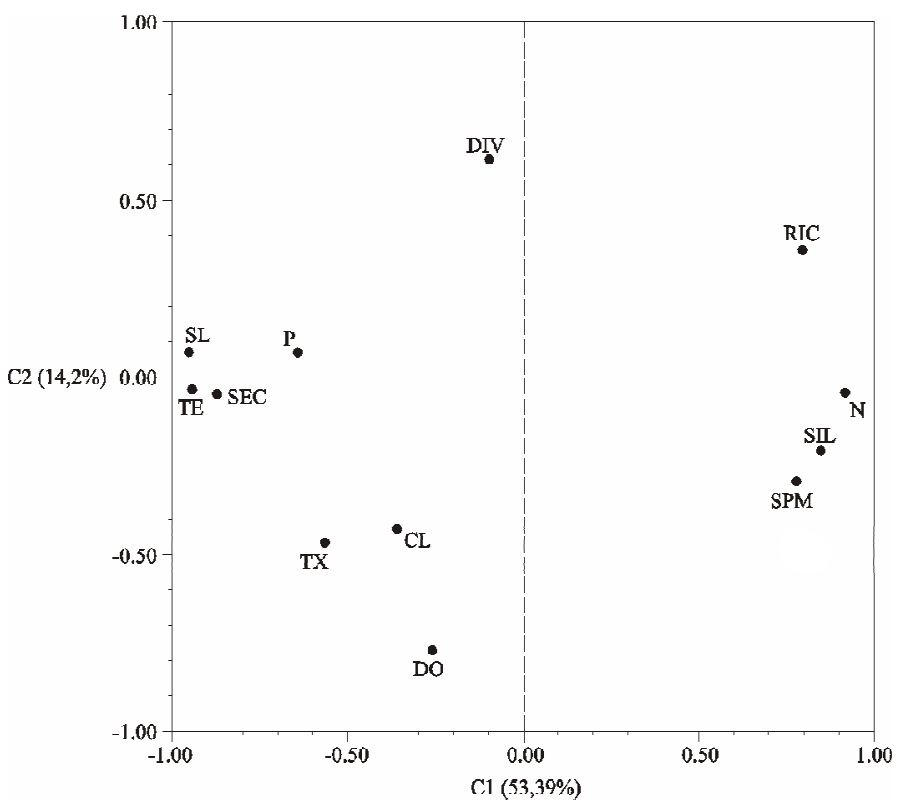

Fig. 6. Principal Component Analysis of the Port Basin of Recife Estuary (Pernambuco, Brazil) during the dry season (January 25-26 and February 0203,2005 ) and rainy season (June $07-08$ and 14-15, 2005). Note: $\mathrm{CL}=$ chlorophyll- $a, \mathrm{DIV}=$ species diversity, $\mathrm{P}=$ phosphate, $\mathrm{SPM}=$ suspended particulate matter, $\mathrm{N}=$ nitrite+nitrate, RIC = number of species, $\mathrm{DO}=$ dissolved oxygen, $\mathrm{SL}=$ salinity, $\mathrm{SIL}=$ silicate, $\mathrm{Tx}=$ oxygen saturation rate, $\mathrm{TE}$ $=$ water temperature and $\mathrm{SEC}=$ water transparency.

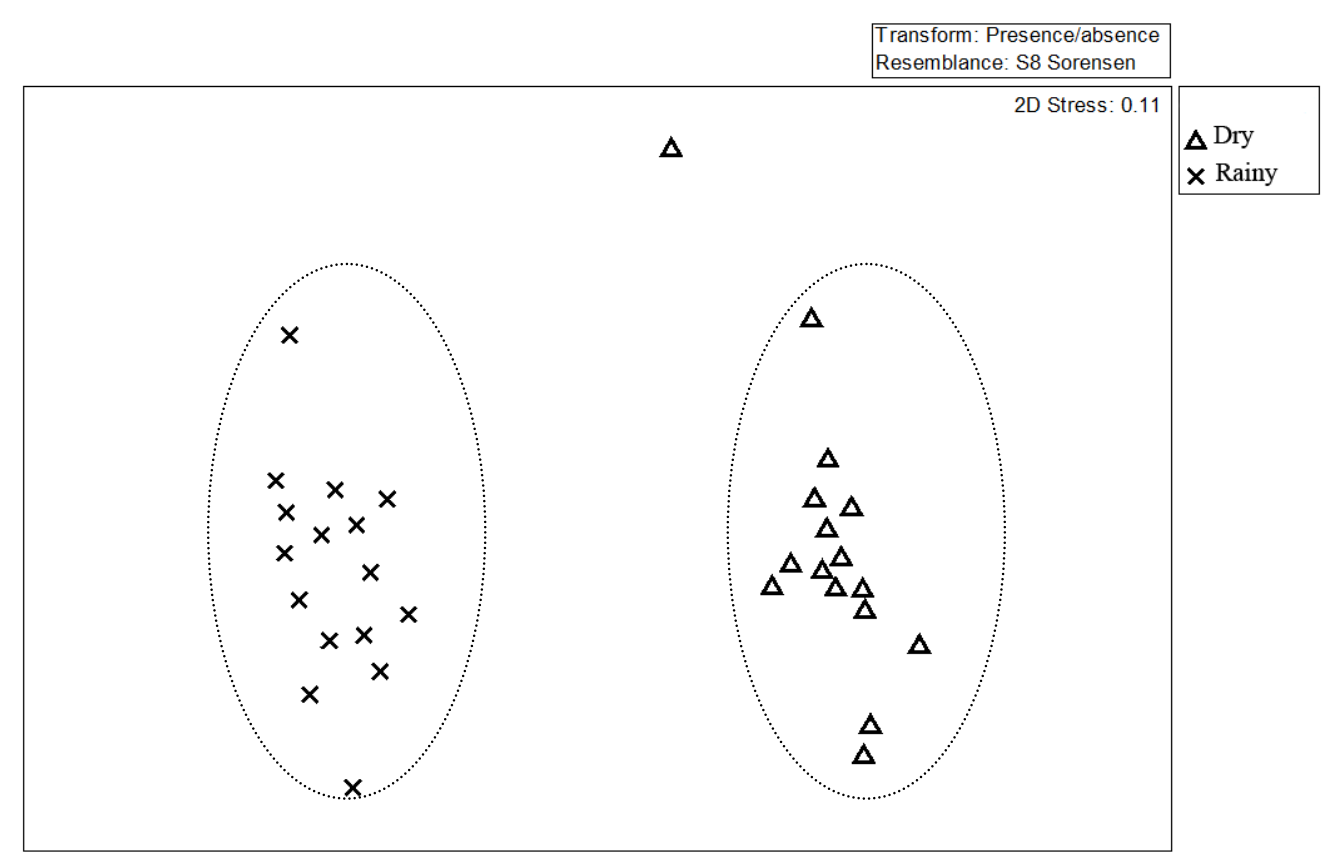

Fig. 7. Multidimensional scale of samples from the Port Basin of Recife Estuary (Pernambuco, Brazil) during the dry season (January 25-26 and February 02-03, 2005) and rainy season (June 07-08 and 14-15, 2005). 
Previous research in the port of Recife and adjacent estuary shows that rainfall, Capibaribe and Beberibe Rivers and Pina Basin run-off affect the harbor area and produce temporal and spatial changes in the dynamics of coastal waters and phytoplankton standing crops (FEITOSA; PASSAVANTE, 1990; RESSURREIÇÃO et al., 1990; FEITOSA et al., 1999). The port of Recife presents features typical of estuarine waters during the rainy season when runoff is high and nutrients are abundant. Later, these nutrients fuel the phytoplankton blooms that commonly occur during the next dry season, when the river discharges decrease appreciably.

These blooms in the dry season bring about conditions of high productivity and create an unbalanced area. In our study, the high chlorophyll- $a$ values were associated with high nutrient loads which enhance the eutrophication recorded in the adjacent Pina Basin (FEITOSA; PASSAVANTE, 1990; FEITOSA et al., 1999; NASCIMENTO et al., 2003) and other coastal environments (VARELA; PREGO, 2003; MELO-MAGALHÃES; KOENING; SANT' ANNA, 2004).

During this study, dissolved oxygen, saturation rate and phosphate showed significant differences with respect to changes in tidal stages, which suggests a great influence of the mixture between river flux and marine waters. Additionally, wind action can cause upwelling in shallow areas, causing resuspension of particulate matter and nutrients (McLUSKY; ELLIOT, 2004). The other physical, chemical and biological parameters showed no significant differences with low variation during the day, probably resulting from the mixing of these water masses.

Although nutrients appear to be available for the production of large quantities of phytoplankton in the study area, maximal production is apparently achieved only during neap tides in the dry season due to greater water transparency and duration of residence. In this time period, phytoplankton displayed an enhanced biomass with chlorophyll- $a$ values up to $55 \mathrm{mg} \mathrm{m}^{-3}$.

According to McLusky and Elliot (2004), three factors limit phytoplankton production: turbidity can limit the penetration of light, shallowness means that blooms may not develop and the growth rate of phytoplankton may be less than the flushing rate of the estuary.

The estuarine area adjacent to Recife Harbor presents eutrophic to hyper-eutrophic conditions. This is typical of urban estuaries with strong anthropogenic impacts (e.g., dredging processes, dumping of municipal sewage), as documented for the Pina Basin (FEITOSA; PASSAVANTE, 1990; FEITOSA et al., 1999; NASCIMENTO et al., 2003) and the Capibaribe River (KOENING et al., 1995). This process has been observed in many other urban areas (e.g., ALMEIDA et al., 2002; ODEBRECHT et al., 2005).

The higher phytoplankton richness in the rainy season was influenced by the input of freshwater species (chlorophytes and cyanobacteria). However, diatoms were the dominant phytoplankton group and some high density species (Helicotheca tamesis, Coscinodiscus centralis, Coscinodiscus kutzingii) were often responsible for the low diversity index. This group of species is predominant in other tropical estuaries under marine influence, probably due to their euryhaline characteristics (ESKINAZI-LEÇA et al., 2000; KOENING et al., 2003; LACERDA et al., 2004; MELO-MAGALHÃES; KOENING; SANT'ANNA, 2004; ROSEVEL DA SILVA et al., 2005; GAMEIRO et al., 2007).

The exceptionally high numbers of chlorophytes and cyanobacteria species (mainly Anabaena, Oscillatoria, Pediastrum, Scenedesmus and Staurastrum) in the rainy season were caused by the intense freshwater flux. This condition and the affinity of these groups with high nutrient concentrations favor their development, instead of the development of other photosynthesizing organisms (GRAHAM; WILCOX, 2000). Chlorophytes were the second group in terms of species richness. In general, this group is the most important component of oligotrophic and eutrophic continental waters (BICUDO; PARRA, 1995).

In our study we observed a great interplay of variables influencing the rate of phytoplankton photosynthesis (e.g., nutrient limitation, light limitation) and factors influencing species composition (e.g., tides, salinity). Rainfall influenced seasonal variability with high inputs of nutrients and sediments. Research into primary production in similar environmental conditions has shown that inputs stimulate phytoplankton growth when light conditions improve and that primary productivity decreases as turbidity increases (FEITOSA; PASSAVANTE, 1991, 1993; RESSURREIÇÃO; PASSAVANTE; MACEDO, 1996; ESKINAZI-LEÇA; KOENING; SILVA-CUNHA, 2000).

When primary production is high, eutrophication can lead to harmful algal blooms in the phytoplankton. Even in this case, total primary production will not necessarily change, but the changes in nutrient concentrations and ratios may influence the species composition of phytoplankton and have profound ecological implications (DAY JR. et al. 1989).

Studies carried out by Marone et al. (2005) close to a port in the Paranaguá Bay suggest that eutrophication processes and their consequences (e.g., oxygen depletion) tend to be more intense during the net heterotrophic rainy period and care should be taken to minimize the effect of the sewage load on the environment, especially during the rainy season. 


\section{ACKNOWLEDGMENTS}

We wish to thank the Coordenação de Aperfeiçoamento de Pessoal de Apoio de Nível Superior (CAPES) for the grant to the first author; Drs. Enide Eskinazi Leça, Maria Luise Koening and Manuel de Jesus Flores Montes for reviewing the paper; Dr. Ralf Schwamborn for his suggestions; Zinaldo Ferreira dos Santos for his important help during field experiments and Mr. José Arnaldo of the Casa de Banho restaurant (Bar do Dique) for his support during our field work.

\section{REFERENCES}

ALMEIDA, M. A.; CUNHA, M. A.; ALCÂNTARA, F. Seasonal change in the proion of bacterial and phytoplankton production along a salinity gradient in shallow estuary. Hydrobiology, v. 475/476, p. 251-262, 2002.

ARAGÃO, J. O. R. A influência dos oceanos Pacífico e Atlântico na dinâmica do tempo e do clima do Nordeste do Brasil. Pp. 287-317. In: ESKINAZI-LEÇA, E.; NEUMANN-LEITÃO, S.; COSTA, M. F. (Org.). Oceanografia - Um cenário tropical. Recife: Bagaço, 2004.

BAIRD, M. E.; EMSLEY, S. M.; MCGLADE, J. M Modelling the interacting effects of nutrient uptake, light capture and temperature on phytoplankton growth. $\mathbf{J}$. Plankt. Res., v. 23, p. 829-840, 2001.

BAUMGARTEN, M. G. Z.; ROCHA, J. M. B.; NIENCHESKI, L. F. H. Manual de analises em oceanografia química. Rio Grande: EDFURG, 1996. $132 \mathrm{p}$.

BICUDO, C. E. M.; PARRA, O. O. Introducción a la biologia y sistemática de las algas continentales. Santiago: Ediciones Universidad de Concepción, 1995.

CUPP, E. E. Marine plankton diatoms of the west coast of North America. Bull. Scripps Inst. Oceanogr., v. 5, p. $1-237,1943$

DAY JR., J. W.; HALL, C. A. J.; KEMP, W. M.; YÁÑEZARANCIBIA, A. Estuarine Ecology. New York: Wiley-Interscience, 1989. 556 p.

DESIKACHARY, T. V. Cyanophyta. New Delhi: Indian Council of Agricultural Research, 1959. $686 \mathrm{p}$.

ESKINAZI-LEÇA, E.; KOENING, M. L.; SILVA-CUNHA M. G. G. O fitoplâncton: estrutura e produtividade. p. 6774. In: BARROS, H. M. ESKINAZI-LECA, E.; MACÊDO, S. J.; LIMA, T. (Ed.). Gerenciamento participativo de estuários e manguezais. Recife: EDUFPE, 2000. $252 \mathrm{p}$.

FEITOSA, F. A. N.; PASSAVANTE, J. Z. O. Variação sazonal da biomassa primária do fitoplâncton da Bacia do Pina (Pernambuco - Brasil). Trabhs oceanogr., Univ.Fed. Pernambuco, v. 21, p. 33-46, 1990.

FEITOSA, F. A. N.; PASSAVANTE, J. Z. O. Variação sazonal da produção primária do fitoplâncton da Bacia do Pina (Pernambuco - Brasil). Trabhs oceanogr. Univ. Fed. Pernambuco, v. 22, p. 65-82, 1991/1993.
FEITOSA, F. A. N.; NASCIMENTO, F. C. R.; COSTA, K. M. P. Distribuição espacial e temporal da biomassa fitoplanctônica relacionada com parâmetros hidrológicos na Bacia do Pina (Pernambuco - Brasil). Trabs oceanogr., Univ. Fed. Pernambuco, v. 27, n. 2, p. 113, 1999.

FERNANDES, L. F.; BRANDINI, F. P. Diatom associations in shelf waters off Paraná State, Southern Brazil: annual variation in relation to environmental factors. Braz. J. Oceanogr., v. 52, n. 1, p. 19-34, 2004.

GAMEIRO, C; CARTAXANA, P.; BROTAS, V. Environmental drivers of phytoplankton distribution and composition in Tagus Estuary, Portugal. Estuar.coast. Shelf Sci., v. 75, p. 21-34, 2007.

GILBERT, P. M. Interactions of top-down and bottom-up control in planktonic nitrogen cycling. Hydrobiologia, v. 363, p.1-12, 1998.

GRAHAM, L. E.; WILCOX, L.W. Algae. New Delhi: Prentice Hall, 2000. 640 p.

GRASSHOFF, K.; EMRHARDT, M.; KREMLING, K. Methods of seawater analysis. 2. ed. New York: Verlag Chemie, $1983.419 \mathrm{p}$.

HUSTEDT, F. Die Kieselalgen Deutschlands, Österreichs und der Schweiz unter Berücksichtigung der übrigen Länder Europas sowie der angrenzenden Meeresgebiete. Kriptogamen-Flora von Deutschlands, Österreichs und der Schweiz, Leipzig, v. 7, pt. 1, p. 920, 1930; pt. 2, p. 845, 1959; pt. 3, p. 816, 1961-1966.

KOENING, M. L.; MACÊDO, S. J.; TRAVASSOS, P. E. P. F.; PASSAVANTE, J. Z. O. Biomassa fitoplanctônica no estuário do rio Capibaribe (Recife - Pernambuco Brasil). Arq. Biol. Tecnol., v. 38, n. 4, p. 1071-1083, 1995

KOENING, M. L.; ESKINAZI LEÇA, E.; NEUMANNLEITÃO, S.; MACEDO, S. J. Impacts of the construction of the (omission ?) of Suape on phytoplankton in the Ipojuca River estuary (Pernambuco-Brazil). Braz. Arch. Biol. Technol., v. 46, n. 1, p. 3-81, 2003.

.KOMÁREK, J.; ANAGNOSTIDIS, K. Cyanoprokaryota. 1. Teil: Chroococcales. Berlim: Spektrum, Akademische Verlag, 2000. (Sübwasserflora von Mitteleuropa; Band 19/1).

LACERDA, S. R.; KOENING, M. L.; NEUMANNLEITÃO, S.; FLORES-MONTES, M. J. Phytoplankton nyctemeral variation at a tropical river estuary (Itamaracá-Pernambuco-Brazil). Braz. J. Biol., v. 64, n.1, p. 81-94, 2004.

LEGENDRE, P.; LEGENDRE, L. Numerical Ecology. Amsterdam: Elsevier Science, 1998.853 p. (Developments in Environmental Modelling, 20).

LEWITUS, A. J.; KOEPFLER, E. T.; MORRIS, J. T. Seasonal variation in the regulation of phytoplankton by nitrogen and grazing in a salt-marsh estuary. Limnology and Oceanography, Baltimore, v. 43, p. 636-646, 1998.

MAFRA JUNIOR, L.; FERNANDES, L. F.; PROENÇA, L. A. O. Harmful algae and toxis in Paranaguá bay, Brazil: bases for monitoring. Braz.J. Oceanogr., v. 54, n. 2-3, p. $107-121,2006$

MAGUIRE, B. Niche response structure and the analytical potentials of its relationship to the habitat. Am. Naturalist, v. 107, n. 954, p. 213-246, 1973. 
MANN, K. H. Ecology of Coastal Waters: A System approach. Oxford: Blackwell Science Publications, 1982. $322 \mathrm{p}$

MARONE, E.; MACHADO, E. C.; LOPES, R. M.; SILVA, E. T. Land-ocean fluxes in the Paranaguá Bay estuarine system, southern Brazil. Braz. J. Oceanogra., v. 53, n. 3-4, p. 169-181, 2005.

McLUSKY, D. S.; ELLIOTT, M. The Estuarine Ecosystem. New York: John Wiley and Sons, 2004.

MELO-MAGALHÃES, E. M; KOENING, M. L.; SANT'ANNA, C. L. Fitoplâncton e variáveis ambientais nos canais do sistema estuarino lagunar Mundaú/Manguaba, Alagoas, Brasil. Hoehnea, , v. 31, n.1, p. 73-86, 2004.

MILLER, C. A.; PENRY, D. L.; GLIBERT, P. M. The impact of trophic interactions on rates of nitrogen regeneration and grazing in Chesapeake Bay. Limnol. Oceanogr., v. 40, p. 1005-1011, 1995.

NASCIMENTO, F. C. R.; MUNIZ, K.; FEITOSA, F. A. N.; ARAÚJO, J. P.; SILVA, R. M. S.; SILVA, G. S.; FLORES MONTES, M. J. Disponibilidade nutricional da Bacia do Pina e rio Tejipió (Recife- PE- Brasil) em relação aos nutrientes e biomassa primária (setembro/2000). Trop. Oceanogr., v. 31, n. 2, p. 149169, 2003.

NEWELL, G. H.; NEWELL, R. Marine plankton: a practical guide. London: Hutchinson Education, 1963. $221 \mathrm{p}$.

ODEBRECHT C. P.; ABREU, O. O.; MÖLLER JR., L. F ; NIENCHESKI, L. A.; PROENÇA-TORGAN, L. C. Dry effects on pelagic propetiers (properties?) in the shallow and turbid Patos lagoon, Brazil. Estuaries, v. 28, p. 675685,2005

PARRA, O. O.; GONZALEZ, M.; DELlAROSSA, V.; RIVERA, P.; ORELLANA, M. Manual taxonomico del fitoplancton de aguas continentales com especial referencia al fitoplancton de Chile. Concepcion: [s.n.], 1982.70 p. (v.1: Cyanophyceae).

PARSONS, T. R.; STRICKLAND, J. D. H. Discussion of spectrophotometric determination of marine plankton pigments, with revised equations of ascertaining chlorophyll $a$ and carotenoids. J. mar. Res., v. 21, n. 3, p. $155-163,1963$.

PÉRAGALLO, H.; PÉRAGALlO, M. Diatomées marines de France et des districts maritimes voisins. Paris: $M$. J. Tempere, 1897-1908.

PRESCOTT, G. W. Algae of the western great lakes area. Duduque: Wm. C. Brown, 1975.

RABOUILLE, C.; MACKENZIE, F. T.; VER, L. M. Influence of the human perturbation on carbon, nitrogen, and oxygen biogeochemical cycles in the global coastal ocean. Geochim. Cosmochim. Acta, v. 65, p.36153641,2001 .

RESH, V. H.; UNZICKER, J. D. Water quality monitoring and aquatic organisms: the importance of species identification. J. Wat. Pollut. Control Fed., v. 47, n. 1, p. 9-19. 1975 .

RESSURREIÇÃO, M. G.; PASSAVANTE， J. Z. O. MACÊDO, S. J. Estudo da plataforma Continental na área do Recife (Brasil): variação sazonal da biomassa fitoplanctônica $\left(08^{\circ} 03^{\prime} 38^{\prime \prime}\right.$ lat. S; $34^{\circ} 42^{\prime} 28^{\prime \prime}$ a $34^{\circ} 52^{\prime} 00^{\prime}$ ' long. W). Trabhs oceanogr., Univ. Fed. Pernambuco, v. 24, p. 39-59, 1990.
RIEGMAN, R.; NOORDELOOS, A. A. M. Size-fractionated uptake of nitrogenous nutrients and carbon by phytoplankton in the North Sea during summer, 1994. Mar. Ecol. Progr. Ser., v. 173, p. 95-106, 1998.

RIEGMAN, R.; FLAMELING, I. A.; NOORDELOOS, A. A. M. Size-fractionated uptake of ammonium, nitrate and urea and phytoplankton growth in the North Sea during spring 1994. Mar. Ecol. Progr. Ser.ies, v. 173, p. 85-94, 1998.

ROHLF, F. J.; FISHER, D. L. Test for hierarchical structure in random data sets. Systematic Zoology, v. 17, p. 407 412, 1968.

ROSEVEL DA SILVA, M.; SILVA-CUNHA, M. G. G.; FEITOSA, F. A. N.; MUNIZ, K. Estrutura da comunidade fitoplânctônica na baía de Tamandaré (Pernambuco, Nordeste do Brasil). Trop. Oceanogr., Recife, v. 33, n.2, p.163-181, 2005.

SHANNON, L. E. A mathematical theory of communication. Bull. of System Tecnology Journal, Curitiba, v. 27, p. 379-423, 1948.

SMAYDA, T. J. Harmful algal blooms: their ecophysiology and general relevance to phytoplankton blooms in the sea. Limnol. Oceanogr., v. 42, p.1137-1153, 1997.

SOROKIN, Y. I. Role of plankton in the turnover of organic matter on the Great Barrier Reef, Australia. Hydrobiologia, v. 308, n. 1, p. 35-44, 1995.

SOURNIA, A. Atlas du phytoplancton marin: introduction, Cyanophycées, Dictyochophycées, Dinophycées et Raphidophycées. Paris: Centre National de la Recherche Scientifique, 1986.

SOURNIA, A. Cycle annuel du phytoplankton et de la production primaire dans les mers tropicales. Mar. Biol., p. 3287-3303, 1969.

SOURNIA, A. Le genre Ceratium (Peridinien planctonique) dans le canal Mozambique: contribution a une révision mondiale. Biologie Marine ., Paris, v.22/23, p. 375-499, 1967.

STRICKLAND, J. D. H.; PARSONS, T. R. A. Manual of Seawater Analysis. Bull. Fish. Res. Bd Canada, v. 125, p. 1-205, 1972.

TAGUSHI, S.; LAWS, E. A. Patterns and causes of temporal variability of the phytoplankton community in Kaneohe Bay, Hawaii. J. Plankt. Res., v. 9, p. 1143-1157, 1987.

TOMAS, C. R. (Ed.). Identifying marine diatoms and dinoflagellates. San Diego: Academic Press, 1996.

UNESCO. International Oceanographic Table. Wormly: Optichrome, 1973.

VARELA, M.; PREGO, R. Hydrography and phytoplankton in an isolated and non-pristine ria(?) area: the A Coruña Harbour (NW Spain). Acta Oecol., v. 24, p. 113-124, 2003.

WETZEL, R. G.; LIKENS, G. E. Limnological analyses. 2. ed. New York: Springer-Verlag, 1991. 391 p.

(Manuscript received 30 July 2009; revised 14 March 2010; accepted 06 May 2010) 